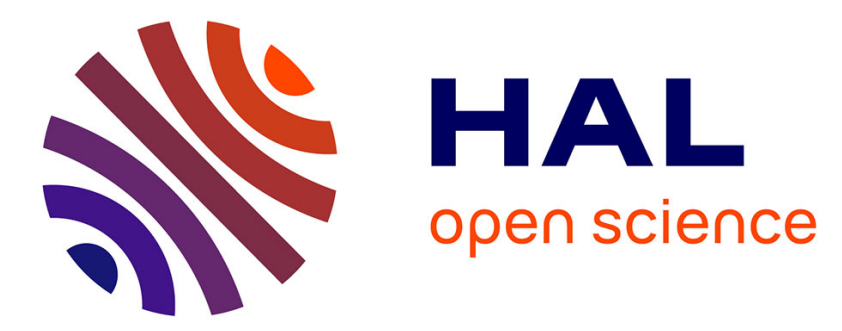

\title{
Error analysis of a dynamic model adaptation procedure for nonlinear hyperbolic equations
}

Clément Cancès, Frédéric Coquel, Edwige Godlewski, Hélène Mathis, Nicolas Seguin

\section{- To cite this version:}

Clément Cancès, Frédéric Coquel, Edwige Godlewski, Hélène Mathis, Nicolas Seguin. Error analysis of a dynamic model adaptation procedure for nonlinear hyperbolic equations. Communications in Mathematical Sciences, 2016, 14 (1), pp.1-30. 10.4310/CMS.2016.v14.n1.a1 . hal-00852101

\section{HAL Id: hal-00852101 https://hal.science/hal-00852101}

Submitted on 20 Aug 2013

HAL is a multi-disciplinary open access archive for the deposit and dissemination of scientific research documents, whether they are published or not. The documents may come from teaching and research institutions in France or abroad, or from public or private research centers.
L'archive ouverte pluridisciplinaire HAL, est destinée au dépôt et à la diffusion de documents scientifiques de niveau recherche, publiés ou non, émanant des établissements d'enseignement et de recherche français ou étrangers, des laboratoires publics ou privés.

\section{(ㄷ)(1) $\$$}

Distributed under a Creative Commons Attribution - NonCommerciall 4.0 International 


\title{
ERROR ANALYSIS OF A DYNAMIC MODEL ADAPTATION PROCEDURE FOR NONLINEAR HYPERBOLIC EQUATIONS
}

\author{
C. CANCÈS, F. COQUEL, E. GODLEWSKI, H. MATHIS, AND N. SEGUIN
}

\begin{abstract}
We propose a dynamic model adaptation method for a nonlinear conservation law coupled with an ordinary differential equation. This model, called the fine model, involves a small time scale and setting this time scale to 0 leads to a classical conservation law, called the coarse model, with a flux which depends on the unknown and on space and time. The dynamic model adaptation consists in detecting the regions where the fine model can be replaced by the coarse one in an automatic way, without deteriorating the accuracy of the result. To do so, we provide an error estimate between the solution of the fine model and the solution of the adaptive method, enabling a sharp control of the different parameters. This estimate rests upon stability results for conservation laws with respect to the flux function. Numerical results are presented at the end and show that our estimate is optimal.

Key-words. Conservation laws, error estimate, model adaptation, thick coupling interface.
\end{abstract}

AMS Subject Classification. 35L65, 35B45, 35B30, 35A35.

\section{Contents}

1. Introduction

2. The models and the dynamic model adaptation

2.1. The fine model

2.2. The coarse model

2.3. Adaptive modeling

3. Quantifying the modeling error linked to adaptation 7

3.1. Estimation of the error $\left\|v_{f}-v_{a}\right\|$

3.2. Quantification of the error $\left\|u_{f}-u_{a}\right\| \quad 11$

4. Application to a model of transport with inertia $\quad 15$

5. Conclusion 17

Appendix A. Stability results for scalar conservation laws $\quad 18$

A.1. Total variation estimates for Kružkov entropy solutions 19

A.2. Stability w.r.t. the flux functions 20

$\begin{array}{ll}\text { References } & 28\end{array}$

\section{INTRODUCTION}

In the context of the simulation of complex fluids, the inner heterogeneities of a flow may lead to use several models with different degrees of accuracy. In an ideal situation, these models can be related one another through asymptotic analysis: singular limits, homogenization, space reduction... A model may be preferred to 
another according to the local features of the flow: for the computations, one aims at using the simplest one without deteriorating the accuracy of the results when compared to those obtained with the finest model. It naturally gives rise to problems of coupling if different models are used in different zones of the computational domain at the same time. Moreover, in the case of transient flows, these zones may evolve in agreement with the structures of the flow. Therefore, we have to tackle the problem of the automatic detection of the best model to use among a given hierarchy of models; this is what we call dynamic model adaptation. The difficulties are twofold:

- how to estimate the decrease of accuracy due to the local use of a coarser model instead of a reference model,

- how to handle the use of different models in different regions of the computational domain.

A byproduct of these two issues is the necessity of estimating the error due to the coupling of the different models. Such estimates are usually called modeling error estimates and go back to the early works of Oden and coworkers [32, 29, 28, 31] for heterogeneous materials, Stein and Ohnimus [30] for Solid Mechanics, and Actis, Szabo and Schwab [1] for laminated plates and shells. In [5], Braack and Ern develop a posteriori error estimators in a general setting in order to equilibrate the modeling error with the numerical error for a global adaptive method. Since these pioneer works, model adaptation has known a large increase of interest, see for instance $[27,2,26,15,16,13]$.

The applications we have in mind here prevents us from following the methodologies proposed in the literature. Indeed, we are interested in compressible fluids with stiff effects such as phase transition, thermal exchanges or drag force in twophase flows, and more generally to nonlinear hyperbolic systems of PDE's. Model adaptation for several of these applications have already been addressed in [25] by the authors, in a more heuristic way due to the complexity of the investigated models. Given a system of balance laws with a stiff source term, we proposed in [25] an error indicator to dynamically replace this model by its equilibrium approximation. To do so, a Chapman-Enskog expansion is performed at the numerical level and we use the first-order corrector as the modeling error indicator. Even if it is impossible to theoretically assess the relevance of this approach, the numerical results are convincing. We then obtain a fully dynamic model adaptation method for nonlinear systems of hyperbolic equations (very similar methods are proposed in $[15,16]$ for kinetics equations and their hydrodynamic limits). In the present paper, we aim at providing theoretical arguments in favor of our approach by using error estimates for nonlinear hyperbolic equations. Due to the limited knowledge in the mathematical study of nonlinear systems of conservation laws, see for instance [14], we have no other choice than restricting ourselves to much simpler models than those investigated in [25]. The "hierarchy of models" we focus on consists of a two equations system made of scalar conservation law together with a stiff ordinary differential equation. The two equations of the fine model (1) may be decoupled in order to simplify the analysis, which is already quite intricate. This leads us to consider the following fine model:

$$
\left\{\begin{array}{l}
\partial_{t} u_{f}(x, t)+\partial_{x} F\left(u_{f}(x, t), v_{f}(x, t)\right)=0 \\
\partial_{t} v_{f}(x, t)=\frac{1}{\tau}\left(v_{\mathrm{eq}}(x, t)-v_{f}(x, t)\right)
\end{array}\right.
$$


where $u_{f}, v_{f}: \mathbb{R} \times \mathbb{R}_{+} \rightarrow \mathbb{R}$ are the unknowns. The functions $F$ and $v_{\text {eq }}$ are assumed to be smooth and the constant $\tau>0$ denotes the characteristic relaxation time and may be considered to be small. As a result, if this time is neglected in the sense $\tau \rightarrow 0$, one obtains the coarse model

$$
\left\{\begin{array}{l}
\partial_{t} u_{c}(x, t)+\partial_{x} F\left(u_{c}(x, t), v_{\mathrm{eq}}(x, t)\right)=0, \\
v_{c}(x, t)=v_{\text {eq }}(x, t)
\end{array}\right.
$$

The dynamic model adaptation consists in replacing (1) by (2) automatically in time and space with a sharp control of the error induced by this replacement and by the coupling at the transition zones between the two models. The procedure leads to the computation of an "adapted solution" $\left(u_{a}, v_{a}\right)$ to be compared to the exact solution $\left(u_{f}, v_{f}\right)$. In practice, our algorithm todefine the solution $\left(u_{a}, v_{a}\right)$ to our dynamic model adaptation relies on a discrete-in-time procedure. To evolves from time $t_{n}$ to time $t_{n+1}=t_{n}+\Delta t_{a}, \Delta t_{a}$ being a given time step, we perform the following computations (we only present in this introduction a simplified and slightly inexact version, see Section 2.3 for the full description of the method):

(1) Assume that $\left(u_{a}, v_{a}\right)\left(t_{n}, \cdot\right)$ is known and let $\Sigma>0$ be a given threshold.

(2) Solve the Cauchy problem for all $x \in \mathbb{R}$

$$
\left\{\begin{array}{l}
\partial_{t} v_{i}(x, t)=\frac{1}{\tau}\left(v_{\mathrm{eq}}(x, t)-v_{i}(x, t)\right), \quad t \in\left[t_{n}, t_{n+1}\right], \\
v_{i}\left(x, t_{n}\right)=v_{a}\left(x, t_{n}\right) .
\end{array}\right.
$$

(3) Define the fine domain by $\Omega_{f}^{(n)}=\left\{x \in \mathbb{R} \mid\left\|v_{i}-v_{\text {eq }}\right\|_{1, \infty}>\Delta t_{a} \Sigma\right\}$.

(4) Computation of $v_{a}$ :

(a) Define a regularized characteristic function $\chi_{\delta} \in \mathcal{C}(\mathbb{R} ;[0,1])$ such that $\chi_{\delta}(x, t)=1$ if $x \in \Omega_{f}^{(n)}, \chi_{\delta}(x, t)=0$ if $d\left(x, \Omega_{f}^{(n)}\right) \geq \delta$ and $\operatorname{TV}\left(\chi_{\delta}\right) \leq$ $\operatorname{TV}\left(\mathbf{1}_{\Omega_{f}^{(n)}}\right)$.

(b) Define

$$
v_{a}=\chi_{\delta} v_{i}+\left(1-\chi_{\delta}\right) v_{\mathrm{eq}} \text { in } \mathbb{R} \times\left[t_{n}, t_{n+1}\right] .
$$

(5) Computation of $u_{a}$ :

solve the scalar conservation law

$$
\partial_{t} u_{a}+\partial_{x} F\left(u_{a}, v_{a}\right)=0
$$

for $x \in \mathbb{R}$ and $t \in\left[t_{n}, t_{n+1}\right]$, with initial datum $u_{a}\left(\cdot, t_{n}\right)$.

(The main difference with the true model adaptation algorithm studied in this paper concerns Step (3); the precise definition of $\Omega_{f}^{(n)}$ is given in Equation (12). Moreover, we have to add the regularity assumptions (14) on $\chi_{\delta}$.)

Let us briefly explain why we have introduced a regularization at step (4) of the above algorithm. The goal of this paper is to perform an error analysis between the solution $\left(u_{a}, v_{a}\right)$ of the model adaptation and the solution $\left(u_{f}, v_{f}\right)$ of the fine model (1) in order to fix the different parameters of the model adaptation algorithm (the time step $\Delta t_{a}$, the threshold $\Sigma$, and the buffer size $\delta$ ) and obtain an error estimate between $\left(u_{a}, v_{a}\right)$ and $\left(u_{f}, v_{f}\right)$. Due to the possibility of exactly computing $v_{f}$ in the fine model (1), the different models rely on scalar conservation laws of the form

$$
\partial_{t} u+\partial_{x} f(u, x, t)=0 .
$$


[-]It is well-known that the classical theory of well-posedness for such equations requires that $f$ is a smooth function of its three variables [21]. It is obviously the same when one aims at deriving error estimates, see $[22,10,24,3,17,8,20,9,12,23]$ and Appendix A.2. The main tool we use is an estimate when considering two conservation laws with different flux functions. This has been studied by Lucier [24], Bouchut and Perthame [3] and recently by Lécureux-Mercier in [23] (see also [12]). We adapt these results in Appendix A.2 to our setting. We then have to pay a special attention to the smoothness w.r.t. (with respect to) $x$ of the underlying problems to solve. Coupling problems with infinitely thin coupling interfaces can be found for instance in $[18,11,19,25]$ but developing error estimates for such problems seems out of reach: to our knowledge, the only example of (numerical) error estimates for a discontinuous flux $f$ w.r.t. $x$ is done in [7] and this result does not apply here. We thus have to consider a regularizing buffer zone for the model coupling, as done by Degond, Dimarco and Mieussens $[15,16]$ and Boutin, Coquel and LeFloch [4]. This explains why we use the regularized characteristic function $\chi_{\delta}$ in (4) instead of the classical characteristic function $\mathbf{1}_{\Omega_{f}^{(n)}}$. Of course, the error estimate between the solution $\left(u_{a}, v_{a}\right)$ of the model adaptation and the solution $\left(u_{f}, v_{f}\right)$ of the fine model (1) blows up when $\delta$ tends to 0 (since $v_{a}$ does not remain smooth), so that we have to carefully calibrate $\delta$ in order to control the error due to the model adaptation.

Remark 1.1. To lead the analysis, we have to restrict ourselves to simple models. One of the consequences is the necessity to solve the fine equation (3) for $v$ in the whole space domain at each time step, so that we cannot hope to gain any efficiency with such a method. However it is the only setting for which it is possible to provide sharp estimates and relations between the different parameters of the model adaptation method. However, we think useful to find a simplified setting where the adaptation method is thoroughly justified.

Let us now present the outline of the paper. In section 2, the fine model and the associated coarse model are described in details, with a special care to the smoothness of the different functions. Then, the discrete-in-time model adaptation algorithm is given. Section 3 first collects the error estimates due to the adaptation, w.r.t. the different parameters of the adaptive algorithm. Using the error analysis developed in Appendix A, we are able to prove Theorem 3.9 where an error estimate between the solution of the fine model (1) and the solution provided by the dynamic model adaptation procedure is given. In the last section, we numerically illustrate the dynamic model adaptation. The fine model corresponds to a transport equation of a chemical component $u_{f}$ with a speed $v_{f}$ which depends on the external medium. With sufficiently small numerical parameters in order to avoid any interaction between the modeling error and the numerical error, we verify that the error estimate is optimal. The final section is devoted to some related ongoing works and possible extensions.

\section{THE MODELS AND THE DYNAMIC MODEL ADAPTATION}


2.1. The fine model. We first consider the solution $\left(u_{f}, v_{f}\right): \mathbb{R} \times \mathbb{R}_{+} \rightarrow \mathbb{R}^{2}$ of the so-called fine model, that is

$$
\left\{\begin{array}{l}
\partial_{t} u_{f}(x, t)+\partial_{x} F\left(u_{f}(x, t), v_{f}(x, t)\right)=0 \\
\partial_{t} v_{f}(x, t)=\frac{1}{\tau}\left(v_{\mathrm{eq}}(x, t)-v_{f}(x, t)\right) \\
u_{f}(x, 0)=u_{0}(x) \\
v_{f}(x, 0)=v_{0}(x)
\end{array}\right.
$$

In the above system, the following assumptions on the data are supposed to be fulfilled.

(i) The flux function $F$ is supposed to belong to $\mathcal{C}^{2}\left(\mathbb{R}^{2} ; \mathbb{R}\right)$, with uniformly bounded first order and second order derivatives. Moreover, we assume that $F(0, v)=0$ for all $v \in \mathbb{R}$.

(ii) The relaxation time $\tau$ is assumed to be constant and strictly positive.

(iii) Concerning the equilibrium state $v_{\text {eq }}$, it is supposed to belong to $\mathcal{C}^{2}\left(\mathbb{R}_{+} \times\right.$ $\left.\mathbb{R}_{+}\right)$, and to be constant outside of the cylinder $(-R, R) \times \mathbb{R}_{+}$for some $R>0$.

(iv) The initial data $v_{0}$ is supposed to belong to $\mathcal{C}^{2}(\mathbb{R})$, and to be equal to $v_{\text {eq }}$ for $|x| \geq R$. As a consequence, $v_{f} \equiv v_{\text {eq }}$ outside of $(-R, R) \times \mathbb{R}_{+}$.

In the system (7), the second equation is linear and decoupled from the first one, so that it can be solved apart. Therefore, $v_{f}$ is explicitly given by: $\forall(x, t) \in \mathbb{R} \times \mathbb{R}_{+}$,

$$
v_{f}(x, t)=v_{0}(x) e^{-\frac{t}{\tau}}+\frac{1}{\tau} \int_{0}^{t} v_{\mathrm{eq}}(x, a) e^{\frac{a-t}{\tau}} d a .
$$

It is easy to check that $v_{f} \in \mathcal{C}^{2}\left(\mathbb{R} \times \mathbb{R}_{+}\right)$is uniformly bounded as well as its first and second order derivatives, so that the function $s \mapsto F\left(s, v_{f}(x, t)\right)$ is regular enough to ensure the existence and the uniqueness of the Kružkov entropy solution $u_{f}$ (see [21]) to the problem

$$
\left\{\begin{array}{l}
\partial_{t} u_{f}+\partial_{x} F\left(u_{f}, v_{f}\right)=0 \quad \text { in } \mathbb{R} \times \mathbb{R}_{+}, \\
u_{f}(\cdot, 0)=u_{0}
\end{array}\right.
$$

In the sequel, we will assume that $u_{0} \in L^{\infty} \cap \mathrm{BV}(\mathbb{R})$, so that, thanks to $[21,12,23]$, the solution $u_{f}$ itself belongs to $L_{\text {loc }}^{\infty} \cap \mathrm{BV}_{\text {loc }}\left(\mathbb{R} \times \mathbb{R}_{+}\right)$. The stability result is recalled in Appendix A (see Theorem A.1).

2.2. The coarse model. Roughly speaking, in the case where $\tau$ is small, $v_{f}$ should be close to $v_{\text {eq }}$. Therefore, a natural coarse model for approximating the solution of (7) consists in

$$
\left\{\begin{array}{l}
\partial_{t} u_{c}(x, t)+\partial_{x} F\left(u_{c}(x, t), v_{\mathrm{eq}}(x, t)\right)=0 \\
u_{c}(x, 0)=u_{0}(x) \\
v_{c}(x, t)=v_{\mathrm{eq}}(x, t)
\end{array}\right.
$$

Here again, due to the regularity of $v_{\text {eq }}$, the problem (9) admits a unique Kružkov entropy solution $u_{c}$ belonging to $L_{\text {loc }}^{\infty} \cap \mathrm{BV}_{\text {loc }}\left(\mathbb{R} \times \mathbb{R}_{+}\right)$for $u_{0} \in L^{\infty} \cap \mathrm{BV}(\mathbb{R})$. 
2.3. Adaptive modeling. We aim now at solving the coarse model (9) in the domain where $u_{c}$ is close to $u_{f}$, and to turn back to the resolution of the system (7) in the zones where $u_{c}$ is not a satisfactory approximation of $u_{f}$. To do so, we will introduce a time-dependent partition of $\mathbb{R}$, i.e:

$$
\mathbb{R}=\bar{\Omega}_{f}(t) \cup \bar{\Omega}_{c}(t), \quad \Omega_{f}(t) \cap \Omega_{c}(t)=\emptyset, \quad \forall t \geq 0 .
$$

In order to define the sets $\Omega_{f}$ and $\Omega_{c}$, we introduce three threshold values $\Sigma>0$, $\Sigma^{\prime}$ and $\Sigma^{\prime \prime}>0$, and a time step $\Delta t_{a}>0$ for the adaptation procedure. For $n \in \mathbb{N}$, we denote by $I_{n}$ the interval $\left[t_{n}, t_{n+1}\right)$, where $t_{k}=k \Delta t_{a}$. We also introduce the size of the regularized buffer zones, denoted $\delta$ in the following.

Let us now describe the adaptation model procedure which defines the functions $\left(u_{a}, v_{a}\right): \mathbb{R} \times \mathbb{R}_{+} \rightarrow \mathbb{R}^{2}$ which approximate the solution $\left(u_{f}, v_{f}\right)$ of the fine model (7):

INITIALIZATION:

Define $\left(u_{a}^{(-1)}, v_{a}^{(-1)}\right)(\cdot, 0)=\left(u_{0}, v_{0}\right)(\cdot)$.

FROM $t_{n}$ TO $t_{n+1}$ :

(1) Define the indicator function $v_{i}^{(n)}: \mathbb{R} \times I_{n} \rightarrow \mathbb{R}$ as the solution of the Cauchy problem

$$
\left\{\begin{array}{l}
\partial_{t} v_{i}^{(n)}=\frac{1}{\tau}\left(v_{\mathrm{eq}}-v_{i}^{(n)}\right) \\
v_{i}^{(n)}\left(\cdot, t_{n}\right)=v_{a}^{(n-1)}\left(\cdot, t_{n}\right)
\end{array}\right.
$$

(2) Define the open subset $\Omega_{f}^{(n)}$ of $\mathbb{R}$ by

$$
\begin{array}{r}
\Omega_{f}^{(n)}=\left\{x \in \mathbb{R}|| v_{\text {eq }}(x, t)-v_{i}^{(n)}(x, t) \mid>\Delta t_{a} \Sigma,\right. \\
\left|\partial_{x} v_{\text {eq }}(x, t)-\partial_{x} v_{i}^{(n)}(x, t)\right|>\Delta t_{a} \Sigma^{\prime}, \\
\text { or } \left.\left|\partial_{x x}^{2} v_{i}^{(n)}\right|>\Sigma^{\prime \prime}, \forall t \in I_{n}\right\},
\end{array}
$$

and $\Omega_{c}^{(n)}=\overbrace{\mathbb{R} \backslash \Omega_{f}^{(n)}}^{0}$.

Define $\Omega_{f}(t)=\Omega_{f}^{(n)}$ and $\Omega_{c}(t)=\Omega_{c}^{(n)}$ for all $t \in I_{n}$.

(3) Define a regularized characteristic function $\chi_{\delta}^{(n)} \in \mathcal{C}^{2}(\mathbb{R} ;[0,1])$ such that

$$
\chi_{\delta}^{(n)}(x)=\left\{\begin{array}{ll}
1 & \text { if } x \in \Omega_{f}^{(n)}, \\
0 & \text { if } d\left(x, \Omega_{f}^{(n)}\right) \geq \delta,
\end{array} \quad \forall x \in \mathbb{R},\right.
$$

such that there exist $\alpha_{1}>0$ and $\alpha_{2}>0$ depending neither on $\Omega_{f}$ nor on $\delta$ such that

$$
\left\|\partial_{x} \chi_{\delta}^{(n)}\right\|_{\infty} \leq \frac{\alpha_{1}}{\delta}, \quad\left\|\partial_{x x}^{2} \chi_{\delta}\right\|_{\infty} \leq \frac{\alpha_{2}}{\delta^{2}}
$$

Notice that such a function $\chi_{\delta}^{(n)}$ always exists. In the sequel, we denote by $\chi_{\delta}: \mathbb{R} \times \mathbb{R}_{+}$the function defined by

$$
\chi_{\delta}(x, t)=\chi_{\delta}^{(n)}(x) \text { if } t \in I_{n} .
$$

(4) Solution to the model coupling problem:

Define $v_{a}^{(n)} \in \mathcal{C}^{2}\left(\mathbb{R} \times I_{n} ; \mathbb{R}\right)$ by

$$
v_{a}^{(n)}=\chi_{\delta}^{(n)} v_{i}^{(n)}+\left(1-\chi_{\delta}^{(n)}\right) v_{\mathrm{eq}} .
$$


Define $u_{a}^{(n)} \in \mathcal{C}\left(\left[t_{n}, t_{n+1}\right] ; L_{l o c}^{1}(\mathbb{R})\right)$ as the unique Kružkov entropy solution of the scalar conservation law

$$
\left\{\begin{array}{l}
\partial_{t} u_{a}^{(n)}+\partial_{x} F\left(u_{a}^{(n)}, v_{a}^{(n)}\right)=0, \\
u_{a}^{(n)}\left(\cdot, t_{n}\right)=u_{a}^{(n-1)}\left(\cdot, t_{n}\right) .
\end{array}\right.
$$

Thanks to this algorithm, we are now able to define the solution of the dynamic model coupling procedure by: for all $t \in I_{n}$, for almost all $x \in \mathbb{R}$,

$$
\left(u_{a}, v_{a}\right)(x, t)=\sum_{n=0}^{\infty}\left(u_{a}^{(n)}, v_{a}^{(n)}\right)(x, t) \mathbf{1}_{I_{n}} .
$$

We also introduce the following global indicator function

$$
v_{i}(\cdot, t)=\sum_{n=0}^{\infty} v_{i}^{(n)}(\cdot, t) \mathbf{1}_{I_{n}} .
$$

Let us note that for all $t \in I_{0}$, we have $v_{i}=v_{f}$, which yields

$$
v_{a}^{(0)}=\chi_{\delta} v_{f}+\left(1-\chi_{\delta}\right) v_{\mathrm{eq}} .
$$

Remark 2.1. It is worth noticing that neither $v_{a}$ nor $v_{i}$ is continuous w.r.t. time due to the adaptation procedure. Nevertheless, the functions are piecewise smooth and right-continuous w.r.t. time, discontinuities appearing only at the ends $\left(t_{k+1}\right)_{k \geq 0}$ of the model adaptation time steps $I_{k}=\left[t_{k}, t_{k+1}\right)$. Therefore, the function $u_{a}$ is uniquely defined. Moreover, in view of Theorem A.1 presented in Appendix (see also [23]), the total variation of $u_{a}^{(n)}(\cdot, t)$ is controlled for all $t \in I_{n}$, so that the application $t \mapsto \mathrm{TV}\left(u_{a}(\cdot, t)\right)$ is locally bounded on $\mathbb{R}_{+}$.

Remark 2.2. Notice that, thanks to the assumption $v_{f} \equiv v_{\mathrm{eq}}$ on $\{|x|>R\} \times \mathbb{R}_{+}$, one has $\Omega_{f}(t) \subset\{|x|<R\}$ for all $t \geq 0$.

\section{QUANTIFYING THE MODELING ERROR LINKED TO ADAPTATION}

We consider an arbitrary finite time horizon $T>0$, and, for the sake of simplicity, we assume that there exists a positive integer $N_{a}$ such that $T=N_{a} \Delta t_{a}$.

\subsection{Estimation of the error $\left\|v_{f}-v_{a}\right\|$.}

Lemma 3.1. There exists $c$ depending only on $\tau$ and $T$ (but not on $\Sigma, \Sigma^{\prime}, \delta$ and $\left.\Delta t_{a}\right)$ such that, for all $n \geq 0$,

$$
\left|v_{a}\left(x, t_{n}\right)-v_{f}\left(x, t_{n}\right)\right| \leq c \Sigma \mathbf{1}_{|x|<R}
$$

Proof. First, in view of Remark 2.2,

$$
v_{a}\left(x, t_{n}\right)=v_{f}\left(x, t_{n}\right) \text { if }|x| \geq R .
$$

Now, fix $x \in(-R, R)$. Clearly, because of the definition of $v_{a}^{(0)}$, one has

$$
\left|v_{a}(x, 0)-v_{f}(x, 0)\right| \leq \Delta t_{a} \Sigma \leq T \Sigma,
$$

so that (19) holds for $n=0$. Now, assume that (19) holds for $n \geq 0$, then

$$
\left|v_{a}\left(x, t_{n+1}\right)-v_{f}\left(x, t_{n+1}\right)\right| \leq \mathcal{A}_{n}+\mathcal{B}_{n}+\mathcal{C}_{n},
$$


where, due to the definition (16) of $v_{a}^{(n)}$ and since $v_{a}\left(x, t_{n+1}\right)=v_{a}^{(n+1)}\left(x, t_{n+1}\right)$, we have set

$$
\begin{aligned}
\mathcal{A}_{n} & =\left|v_{a}^{(n+1)}\left(x, t_{n+1}\right)-v_{i}^{(n+1)}\left(x, t_{n+1}\right)\right|, \\
\mathcal{B}_{n} & =\left|v_{a}^{(n)}\left(x, t_{n+1}\right)-v_{i}^{(n)}\left(x, t_{n+1}\right)\right| \\
& =\left(1-\chi_{\delta}\left(x, t_{n+1}\right)\right)\left|v_{\mathrm{eq}}\left(x, t_{n+1}\right)-v_{i}^{(n)}\left(x, t_{n+1}\right)\right|, \\
\mathcal{C}_{n} & =\left|v_{i}^{(n)}\left(x, t_{n+1}\right)-v_{f}\left(x, t_{n+1}\right)\right| .
\end{aligned}
$$

Bearing in mind the definitions of $\Omega_{f}^{(n+1)}$ and $\Omega_{f}^{(n)}$, we have

$$
\mathcal{A}_{n} \leq \Delta t_{a} \Sigma \quad \text { and } \quad \mathcal{B}_{n} \leq \Delta t_{a} \Sigma \text {. }
$$

On the other hand, using the fact that $v_{i}^{(n)}(x, \cdot)$ and $v_{f}(x, \cdot)$ satisfy the same linear ODE on $I_{n}$ for different initial data, $w^{(n)}(x, \cdot):=v_{i}^{(n)}(x, \cdot)-v_{f}(x, \cdot)$ is the solution on $I_{n}$ of

$$
\left\{\begin{array}{l}
\partial_{t} w^{(n)}+\frac{1}{\tau} w^{(n)}=0, \\
w^{(n)}\left(x, t_{n}\right)=v_{a}\left(x, t_{n}\right)-v_{f}\left(x, t_{n}\right) .
\end{array}\right.
$$

Therefore,

$$
\mathcal{C}_{n}=e^{-\Delta t_{a} / \tau}\left|v_{a}\left(x, t_{n}\right)-v_{f}\left(x, t_{n}\right)\right| .
$$

In view of (21), (22) and (23), we obtain that

$$
\left|v_{a}\left(x, t_{n+1}\right)-v_{f}\left(x, t_{n+1}\right)\right| \leq 2 \Delta t_{a} \Sigma+e^{-\Delta t_{a} / \tau}\left|v_{a}\left(x, t_{n}\right)-v_{f}\left(x, t_{n}\right)\right|,
$$

yielding by induction that

$$
\left|v_{a}\left(x, t_{n}\right)-v_{f}\left(x, t_{n}\right)\right| \leq \frac{2 \Delta t_{a}}{1-e^{-\Delta t_{a} / \tau}} \Sigma+e^{-n \Delta t_{a} / \tau}\left|v_{a}(x, 0)-v_{f}(x, 0)\right| .
$$

It only remains to check that the function $t \mapsto \frac{2 t}{1-e^{-t / \tau}}$ is increasing, so that, since $\Delta t_{a} \leq T$

$$
\left|v_{a}\left(x, t_{n}\right)-v_{f}\left(x, t_{n}\right)\right| \leq \frac{2 T}{1-e^{-T / \tau}} \Sigma+\left|v_{a}(x, 0)-v_{f}(x, 0)\right| .
$$

We conclude by using (20).

Lemma 3.2. There exists $c$ depending only on $\tau$ and $T$ (but not on $\Sigma, \Sigma^{\prime}, \delta$ and $\left.\Delta t_{a}\right)$ such that

$$
\left|v_{a}(x, t)-v_{f}(x, t)\right| \leq c \Sigma \mathbf{1}_{|x|<R}(x), \quad \forall(x, t) \in \mathbb{R} \times[0, T) .
$$

Proof. In the case $t=T$, then (24) is nothing but (19) with $n=N_{a}$. Assume now that $t \in[0, T)$, then there exists a unique $n \in\{0, \ldots, N-1\}$ such that $t \in I_{n}$. The triangular inequality yields

$$
\left|v_{a}(x, t)-v_{f}(x, t)\right| \leq\left|v_{a}(x, t)-v_{i}(x, t)\right|+\left|v_{i}(x, t)-v_{f}(x, t)\right| .
$$

The first term of the right-hand side is controlled by $\Delta t_{a} \Sigma$, while the second one is controlled by

$$
\begin{aligned}
\left|v_{i}(x, t)-v_{f}(x, t)\right| & \leq e^{-\left(t-t_{n}\right) / \tau}\left|v_{a}^{(n-1)}\left(x, t_{n}\right)-v_{f}\left(x, t_{n}\right)\right| \\
& \leq e^{-\left(t-t_{n}\right) / \tau}\left(\mathcal{B}_{n-1}+\mathcal{C}_{n-1}\right),
\end{aligned}
$$

where the quantities $\mathcal{B}_{n-1}$ and $\mathcal{C}_{n-1}$ have been introduced and controlled in the proof of Lemma 3.1. 
The proof of Lemmas 3.1 and 3.2 relies on two arguments, namely

(1) $v_{f}$ and $v_{i}$ are solutions of the same contractive ODE with different initial data;

(2) the error between $v_{a}$ and $v_{i}$ is controlled thanks to the adaptation procedure.

These two arguments still hold when one considers the space derivatives $\partial_{x} v_{a}, \partial_{x} v_{f}$ and $\partial_{x} v_{i}$ instead of the functions $v_{a}, v_{f}$ and $v_{i}$, but one has to take care of the perturbation introduced by the buffer of size $\delta$. This leads to the following two lemmas.

Lemma 3.3. There exists $c$ depending only on $\tau$ and $T$ (but not on $\Sigma, \Sigma^{\prime}, \delta$ and $\left.\Delta t_{a}\right)$ such that, for all $n \in\left\{0, \ldots, N_{a}\right\}$,

$$
\left|\partial_{x} v_{a}\left(x, t_{n}\right)-\partial_{x} v_{f}\left(x, t_{n}\right)\right| \leq c\left(\Sigma^{\prime}+\frac{\Sigma}{\delta}\right) \mathbf{1}_{|x|<R}(x), \quad \forall x \in \mathbb{R} .
$$

Proof. First, we use again Remark 2.2 to claim that, for all $t \geq 0$ and for all $x$ such that $|x| \geq R$, one has

$$
\partial_{x} v_{a}(x, t)=\partial_{x} v_{f}(x, t) .
$$

Consider now the case $x \in(-R, R)$. The definition (18) of $v_{a}^{(0)}$ provides that

$$
v_{a}(x, 0)-v_{f}(x, 0)=\left(1-\chi_{\delta}(x, 0)\right)\left(v_{\mathrm{eq}}(x, 0)-v_{f}(x, 0)\right),
$$

so that, in view of (13) and (14), one has

$$
\begin{aligned}
& \left|\partial_{x} v_{a}(x, 0)-\partial_{x} v_{f}(x, 0)\right| \\
& \quad \leq\left(\left|\partial_{x} v_{\mathrm{eq}}(x, 0)-\partial_{x} v_{f}(x, 0)\right|+\frac{\alpha_{1}}{\delta}\left|v_{\mathrm{eq}}(x, 0)-v_{f}(x, 0)\right|\right) \mathbf{1}_{\Omega_{c}^{(0)}(x)} .
\end{aligned}
$$

It follows from the definition of $\Omega_{f}^{(0)}$ that

$$
\left|\partial_{x} v_{a}^{(0)}(x, 0)-\partial_{x} v_{f}(x, 0)\right| \leq \Delta t_{a}\left(\Sigma^{\prime}+\frac{\alpha_{1} \Sigma}{\delta}\right) \leq c\left(\Sigma^{\prime}+\frac{\Sigma}{\delta}\right) .
$$

Now, fix $n \in\left\{0, \ldots, N_{a}-1\right\}$, then, since $v_{i}^{(n+1)}\left(\cdot, t_{n+1}\right) \equiv v_{a}^{(n)}\left(\cdot, t_{n+1}\right)$, we have

$$
\left|\partial_{x} v_{a}\left(x, t_{n+1}\right)-\partial_{x} v_{f}\left(x, t_{n+1}\right)\right| \leq \mathcal{D}_{n}+\mathcal{E}_{n}+\mathcal{F}_{n},
$$

where we have set

$$
\begin{aligned}
\mathcal{D}_{n} & =\left|\partial_{x}\left(v_{a}^{(n+1)}-v_{i}^{(n+1)}\right)\left(x, t_{n+1}\right)\right|, \\
\mathcal{E}_{n} & =\left|\partial_{x}\left(v_{a}^{(n)}-v_{i}^{(n)}\right)\left(x, t_{n+1}\right)\right|, \\
\mathcal{F}_{n} & =\left|\partial_{x}\left(v_{i}^{(n)}-v_{f}\right)\left(x, t_{n+1}\right)\right| .
\end{aligned}
$$

In view of the definition (16) of $v_{a}^{(n+1)}$, one has

$$
v_{a}^{(n+1)}-v_{i}^{(n+1)}=\left(1-\chi_{\delta}\right)\left(v_{\mathrm{eq}}-v_{i}^{(n+1)}\right) \quad \text { on } \mathbb{R} \times I_{n+1},
$$

so that, due to the definitions of $\Omega_{f}^{(n+1)}$ and $\chi_{\delta}$, we obtain that

$$
\mathcal{D}_{n} \leq \Delta t_{a}\left(\Sigma^{\prime}+\frac{\alpha_{1} \Sigma}{\delta}\right) \mathbf{1}_{\Omega_{c}^{(n+1)}}(x) .
$$

Similarly,

$$
\mathcal{E}_{n} \leq \Delta t_{a}\left(\Sigma^{\prime}+\frac{\alpha_{1} \Sigma}{\delta}\right) \mathbf{1}_{\Omega_{c}^{(n)}}(x)
$$


In order to control $\mathcal{F}_{n}$, we notice that $z^{(n)}:=\partial_{x} v_{i}^{(n)}-\partial_{x} v_{f}^{(n)}$ is the solution on $I_{n}$ of

$$
\left\{\begin{array}{l}
\partial_{t} z^{(n)}+\frac{1}{\tau} z^{(n)}=0, \\
z^{(n)}\left(x, t_{n}\right)=\partial_{x} v_{a}\left(x, t_{n}\right)-\partial_{x} v_{f}\left(x, t_{n}\right),
\end{array}\right.
$$

so that, as in the proof of Lemma 3.1, we obtain that

$$
\mathcal{F}_{n} \leq e^{-\Delta t_{a} / \tau}\left|\partial_{x} v_{a}\left(x, t_{n}\right)-\partial_{x} v_{f}\left(x, t_{n}\right)\right| .
$$

We conclude by mimicking the induction detailed in the proof of Lemma 3.1.

We derive the following lemma, whose proof is left to the reader, from Lemma 3.3 by adapting the proof of Lemma 3.2.

Lemma 3.4. There exists $c$ depending only on $\tau$ and $T$ (but not on $\Sigma, \Sigma^{\prime}, \delta$ and $\left.\Delta t_{a}\right)$ such that

$$
\left|\partial_{x} v_{a}(x, t)-\partial_{x} v_{f}(x, t)\right| \leq c\left(\Sigma^{\prime}+\frac{\Sigma}{\delta}\right) \mathbf{1}_{|x|<R}(x) \quad \forall(x, t) \in \mathbb{R} \times[0, T) .
$$

The last estimate we need concerns the second order space derivative of $v_{a}$.

Lemma 3.5. There exists $c$ depending only on $v_{\mathrm{eq}}, v_{f}$ and $T$ such that

$$
\left|\partial_{x x}^{2} v_{a}(x, t)\right| \leq c\left(1+\Sigma^{\prime \prime}+\frac{\Sigma}{\delta^{2}}+\frac{\Sigma^{\prime}}{\delta}\right), \quad \forall(x, t) \in \mathbb{R} \times[0, T) .
$$

Proof. Here again, the proof is based on an induction argument. For $t \in I_{0}$, it follows from (18) that

$$
\partial_{x x}^{2} v_{a}^{(0)}=\chi_{\delta} \partial_{x x}^{2} v_{f}+\left(1-\chi_{\delta}\right) \partial_{x x}^{2} v_{\mathrm{eq}}+2 \partial_{x}\left(v_{f}-v_{\mathrm{eq}}\right) \partial_{x} \chi_{\delta}+\left(v_{f}-v_{\mathrm{eq}}\right) \partial_{x x}^{2} \chi_{\delta} .
$$

In view of the definition (15) and of the properties (14) of the function $\chi_{\delta}$, we get that

$$
\left|\partial_{x x}^{2} v_{a}^{(0)}\right| \leq \max \left\{\left|\partial_{x x}^{2} v_{f}\right|,\left|\partial_{x x}^{2} v_{\mathrm{eq}}\right|\right\}+\frac{2 \alpha_{1} \Sigma^{\prime}}{\delta}+\frac{\alpha_{2} \Sigma}{\delta^{2}} \quad \text { on } \mathbb{R} \times\left[0, \Delta t_{a}\right] .
$$

In particular, due to the regularity of $v_{f}$ and $v_{\text {eq }}$, the relation (31) holds for $(x, t) \in$ $\mathbb{R} \times I_{0}$ for $c=c_{0}$ defined by

$$
c_{0}=\max \left\{\left\|\partial_{x x}^{2} v_{f}\right\|_{L^{\infty}\left(\mathbb{R} \times I_{0}\right)},\left\|\partial_{x x}^{2} v_{\mathrm{eq}}\right\|_{L^{\infty}\left(\mathbb{R} \times I_{0}\right)}, 2 \alpha_{1}, \alpha_{2}\right\} .
$$

Now, fix $n \geq 1$, and assume that there exists $c_{n-1}$ such that

$$
\left|\partial_{x x}^{2} v_{a}^{(n-1)}\left(x, t^{n}\right)\right|=\left|\partial_{x x}^{2} v_{i}^{(n)}\left(x, t^{n}\right)\right| \leq c_{n-1}\left(1+\Sigma^{\prime \prime}+\frac{\Sigma}{\delta^{2}}+\frac{\Sigma^{\prime}}{\delta}\right) .
$$

Then, for all $x \in \mathbb{R}$, the function $t \mapsto \partial_{x x}^{2} v_{i}^{(n)}(x, t)$ is the solution of the linear ODE

$$
\left\{\begin{array}{l}
\partial_{t} \partial_{x x}^{2} v_{i}^{(n)}(x, t)+\frac{1}{\tau} \partial_{x x}^{2} v_{i}^{(n)}(x, t)=\frac{1}{\tau} \partial_{x x}^{2} v_{\mathrm{eq}}(x, t), \\
\partial_{x x}^{2} v_{i}^{(n)}\left(x, t_{n}\right)=\partial_{x x}^{2} v_{a}^{(n-1)}\left(x, t_{n}\right) .
\end{array}\right.
$$

In particular, for all $t \in \bar{I}_{n}$, one has

$$
\left|\partial_{x x}^{2} v_{i}^{(n)}(x, t)\right| \leq\left|\partial_{x x}^{2} v_{i}^{(n)}\left(x, t^{n}\right)\right| \leq c_{n-1}\left(1+\Sigma^{\prime \prime}+\frac{\Sigma}{\delta^{2}}+\frac{\Sigma^{\prime}}{\delta}\right) .
$$

Recall that the function $v_{a}^{(n)}$ is then defined by (16), so that, on $\mathbb{R} \times \bar{I}_{n}$, one has

$$
\begin{aligned}
\partial_{x x}^{2} v_{a}^{(n)}= & \chi_{\delta} \partial_{x x}^{2} v_{i}^{(n)}+\left(1-\chi_{\delta}\right) \partial_{x x}^{2} v_{\mathrm{eq}} \\
& +2 \partial_{x}\left(v_{i}^{(n)}-v_{\mathrm{eq}}\right) \partial_{x} \chi_{\delta}+\left(v_{i}^{(n)}-v_{\mathrm{eq}}\right) \partial_{x x}^{2} \chi_{\delta} .
\end{aligned}
$$


Since $v_{a}^{(n)} \equiv v_{i}^{(n)}$ in $\Omega_{f}^{(n)} \times \bar{I}_{n}$, one gets directly from (34) that

$$
\left|\partial_{x x}^{2} v_{a}^{(n)}(x, t)\right| \leq c_{n-1}\left(1+\Sigma^{\prime \prime}+\frac{\Sigma}{\delta^{2}}+\frac{\Sigma^{\prime}}{\delta}\right), \quad \forall(x, t) \in \Omega_{f}^{(n)} \times \bar{I}_{n} .
$$

Now, for $(x, t) \in \overline{\Omega_{c}^{(n)} \times I_{n}}$, one has

$$
\left|\partial_{x x}^{2} v_{i}^{(n)}\right| \leq \Sigma^{\prime \prime}, \quad\left|\partial_{x}\left(v_{i}^{(n)}-v_{\mathrm{eq}}\right)\right| \leq \Sigma^{\prime}, \quad \text { and } \quad\left|v_{i}^{(n)}-v_{\mathrm{eq}}\right| \leq \Sigma .
$$

Therefore, using again (14) in (35), we obtain

$$
\left|\partial_{x x}^{2} v_{a}^{(n)}(x, t)\right| \leq\left\|\partial_{x x}^{2} v_{\text {eq }}\right\|_{\infty}+\Sigma^{\prime \prime}+\frac{2 \alpha_{1} \Sigma^{\prime}}{\delta}+\frac{\alpha_{2} \Sigma}{\delta^{2}}, \quad \forall(x, t) \in \overline{\Omega_{c}^{(n)} \times I_{n}} .
$$

In particular, it follows from (36) and (37) that

$$
\left|\partial_{x x}^{2} v_{a}^{(n)}(x, t)\right| \leq c_{n}\left(1+\Sigma^{\prime \prime}+\frac{\Sigma}{\delta^{2}}+\frac{\Sigma^{\prime}}{\delta}\right)
$$

where

$$
c_{n}=\max \left\{c_{n-1},\left\|\partial_{x x}^{2} v_{\mathrm{eq}}\right\|_{L^{\infty}(\mathbb{R} \times(0, T))}, 2 \alpha_{1}, \alpha_{2}\right\} .
$$

In view of the definition (33) of $c_{0}$, we obtain by a straightforward induction that (31) holds with

$$
c=\max \left\{\left\|\partial_{x x}^{2} v_{f}\right\|_{L^{\infty}(\mathbb{R} \times(0, T))},\left\|\partial_{x x}^{2} v_{\mathrm{eq}}\right\|_{L^{\infty}(\mathbb{R} \times(0, T))}, 2 \alpha_{1}, \alpha_{2}\right\} .
$$

3.2. Quantification of the error $\left\|u_{f}-u_{a}\right\|$. In this section, our goal is to quantify the error produced by the adaptation procedure described in Section 2.3. To do so, we will overestimate

$$
\left\|u_{a}-u_{f}\right\|_{C\left([0, T], L^{1}(\mathbb{R})\right)},
$$

where $T$ is an arbitrary final time, thanks to quantities depending on $\Sigma, \Sigma^{\prime}$ and $\delta$. Then, for a suitable choice of these quantities, we will guaranty that the modeling error (38) can be enforced to remain as small as desired.

Setting

$$
f:(s, x, t) \in \mathbb{R} \times \mathbb{R} \times \mathbb{R}_{+} \mapsto f(s, x, t)=F\left(s, v_{a}(x, t)\right)
$$

and

$$
g:(s, x, t) \in \mathbb{R} \times \mathbb{R} \times \mathbb{R}_{+} \mapsto g(s, x, t)=F\left(s, v_{f}(x, t)\right),
$$

the function $u_{a}$ is then defined as the unique entropy solution to the problem

$$
\begin{cases}\partial_{t} u_{a}+\partial_{x} f\left(u_{a}\right)=0 & \text { in } \mathbb{R} \times(0, \infty), \\ u_{a}(\cdot, 0)=u_{0} & \text { in } \mathbb{R},\end{cases}
$$

while $u_{f}$ can be seen as the unique entropy solution to the problem

$$
\begin{cases}\partial_{t} u_{f}+\partial_{x} g\left(u_{f}\right)=0 & \text { in } \mathbb{R} \times(0, \infty), \\ u_{f}(\cdot, 0)=u_{0} & \text { in } \mathbb{R} .\end{cases}
$$

Therefore, in order to quantify the difference between $u_{a}$ and $u_{f}$, we will use a stability result w.r.t. to the flux function established in Appendix.

Using Theorem A.3, we obtain that for all $t^{\star}>0$, one has

$$
\left\|u_{a}\left(\cdot, t^{\star}\right)-u_{f}\left(\cdot, t^{\star}\right)\right\|_{L^{1}(\mathbb{R})} \leq \inf _{\epsilon>0}\left(\epsilon C_{1}+\frac{C_{2}}{\epsilon}+C_{3}\right),
$$


where, in view of the definition of the functions $f$ and $g$, we have

$$
\begin{aligned}
C_{1}= & \alpha+\beta\left\|\partial_{s} \partial_{x} f\right\|_{\infty} \\
& +3 \int_{0}^{t^{\star}} \sup _{s \in \mathbb{R}}\left\|\partial_{x x}^{2} f(s, \cdot, t)\right\|_{\infty} d t, \\
C_{2}= & 2 \int_{0}^{t^{\star}} \int_{\mathbb{R}}\|(f-g)(\cdot, x, t)\|_{\infty} d x d t, \\
C_{3}= & \int_{0}^{t^{\star}} \int_{\mathbb{R}}\left\|\partial_{x}(f-g)(\cdot, x, t)\right\|_{\infty} d x d t .
\end{aligned}
$$

In the definition (42) of $C_{1}$, the quantities $\alpha$ and $\beta$ depend only on $t^{\star}$ (in an increasing way), $u_{0}, F$ and on the fine solution $v_{f}$, but not on the adaptation procedure. They are made explicit in Appendix A.2.

\section{Remark 3.1.}

(1) Theorem A.3 gives a localized in space error estimate. Nevertheless, since the flux functions $s \mapsto F\left(s, v_{a}(x, t)\right)$ and $s \mapsto F\left(s, v_{f}(x, t)\right)$ coincide on $\{|x|>R\} \times \mathbb{R}_{+}$, the contribution of the flux functions variation is located on a finite measure space. Then, the error produced by the adaptation procedure travels with a speed lower or equal to $\left\|\partial_{u} F\right\|_{\infty}$, so that after a time $t$, the functions $u_{f}(\cdot, t)$ and $u_{a}(\cdot, t)$ may differ only on $\{|x|<$ $\left.R+t\left\|\partial_{u} F\right\|_{\infty}\right\}$. Therefore, considering the $L^{1}$-norm on the full space $\mathbb{R}$ is meaningful in (41).

(2) Because of the previous point, the integrals w.r.t. the space variable appearing in (42)-(44) can be considered on the full $\mathbb{R}$. As a consequence, the integration domains do not depend on $\epsilon$ as it is the case in the more general case presented in Appendix. Therefore, the quantities defined as $C_{i}^{\epsilon}$ in the Appendix do not depend on $\epsilon$ in the present case. This justifies the fact that we denote them by $C_{i}$, without $\epsilon$.

Lemma 3.6. There exists $c>0$ depending only on the data $F, u_{0}, v_{f}, v_{\mathrm{eq}}$ and in an increasing way of $t^{\star}$ (but not on the adaptation procedure) such that

$$
C_{1} \leq c\left(1+\Sigma^{\prime \prime}+\Sigma^{\prime}+\frac{\Sigma}{\delta}+\left(\Sigma^{\prime}\right)^{2}+\frac{\Sigma^{2}}{\delta^{2}}+\frac{\Sigma^{\prime}}{\delta}+\frac{\Sigma}{\delta^{2}}\right) .
$$

Proof. Bearing in mind the definition (39) of the flux function $f$, we obtain that

$$
\partial_{s} \partial_{x} f(s, x, t)=\partial_{s} \partial_{v} F\left(s, v_{a}(x, t)\right) \partial_{x} v_{a}(x, t),
$$

so that

$$
\left\|\partial_{s} \partial_{x} f\right\|_{\infty} \leq\left\|\partial_{s} \partial_{v} F\right\|_{\infty}\left(\left\|\partial_{x} v_{f}\right\|_{\infty}+\left\|\partial_{x}\left(v_{a}-v_{f}\right)\right\|_{\infty}\right) .
$$

In view of Lemma 3.4, we deduce that

$$
\left\|\partial_{s} \partial_{x} f\right\|_{\infty} \leq\left\|\partial_{u} \partial_{v} F\right\|_{\infty}\left(\left\|\partial_{x} v_{f}\right\|_{\infty}+\Sigma^{\prime}+\frac{2}{\delta} \Sigma\right) .
$$

Similarly, we have

$$
\partial_{x x}^{2} f(u, x, t)=\partial_{v v}^{2} F\left(u, v_{a}(x, t)\right)\left(\partial_{x} v_{f}(x, t)\right)^{2}+\partial_{v} F\left(u, v_{a}(x, t)\right) \partial_{x x}^{2} v_{a},
$$


so that

$$
\begin{aligned}
\sup _{s \in \mathbb{R}}\left\|\partial_{x x}^{2} f(s, \cdot, t)\right\| \leq & \left\|\partial_{v v}^{2} F\right\|_{\infty}\left(\left\|\partial_{x} v_{f}\right\|_{\infty}+\left\|\partial_{x}\left(v_{a}-v_{f}\right)\right\|_{\infty}\right)^{2} \\
& +\left\|\partial_{v} F\right\|_{\infty}\left\|\partial_{x x}^{2} v_{a}(\cdot, t)\right\|_{\infty} .
\end{aligned}
$$

Using Lemmas 3.4 and 3.5, we deduce from the fact that $\left(\sum_{i=1}^{k} a_{i}\right)^{2} \leq k \sum_{i=1}^{k}\left(a_{i}\right)^{2}$ that

$$
\begin{aligned}
\sup _{s \in \mathbb{R}}\left\|\partial_{x x}^{2} f(s, \cdot, t)\right\| \leq & 3\left\|\partial_{v v}^{2} F\right\|_{\infty}\left(\left\|\partial_{x} v_{f}\right\|_{\infty}^{2}+\left(\Sigma^{\prime}\right)^{2}+\frac{1}{\delta^{2}} \Sigma^{2}\right) \\
& +\left\|\partial_{v} F\right\|_{\infty} c\left(1+\Sigma^{\prime \prime}+\frac{\Sigma^{\prime}}{\delta}+\frac{\Sigma}{\delta^{2}}\right) .
\end{aligned}
$$

Hence, denoting by $c$ a generic quantity depending only on the data $F, u_{0}, v_{f}$, $v_{\text {eq }}$ and in an increasing way of $t^{\star}$, we deduce from (42), (46) and (47) that (45) holds.

Lemma 3.7. There exists $c$ depending only on the data $F, R$ and $t^{\star}$ in an increasing way (but not on the adaptation procedure) such that

$$
C_{2} \leq c \Sigma
$$

Proof. In view of the regularity of $F$ and of Lemma 3.2, we have

$$
|f-g|=\left|F\left(s, v_{a}\right)-F\left(s, v_{f}\right)\right| \leq\left\|\partial_{v} F\right\|_{\infty}\left|v_{f}-v_{a}\right| \leq\left\|\partial_{v} F\right\|_{\infty} \Sigma \mathbf{1}_{(-R, R)} .
$$

Estimate (48) then follows directly from the definition (43) of $C_{2}$.

Lemma 3.8. There exists $c$ depending only on the data $F, v_{f}, R$ and in an increasing way of $t^{\star}$ (but not on the adaptation procedure) such that

$$
C_{3} \leq c\left(\Sigma+\Sigma^{\prime}+\frac{\Sigma}{\delta}\right)
$$

Proof. For all $s \in \mathbb{R}$, one has

$$
\partial_{x} f(s, x, t)=\partial_{x} F\left(s, v_{a}(x, t)\right)=\partial_{v} F\left(s, v_{a}(x, t)\right) \partial_{x} v_{a}(x, t) .
$$

Similarly,

$$
\begin{aligned}
& \partial_{x} g(s, x, t)=\partial_{v} F\left(s, v_{f}(x, t)\right) \partial_{x} v_{f}(x, t) \\
& \quad=\partial_{v} F\left(s, v_{a}(x, t)\right) \partial_{x} v_{f}(x, t)+\left[\partial_{v} F\left(s, v_{a}(x, t)\right)-\partial_{v} F\left(s, v_{a}(x, t)\right)\right] \partial_{x} v_{f}(x, t) .
\end{aligned}
$$

Therefore,

$$
\left|\partial_{x}(f-g)\right| \leq\left\|\partial_{v} F\right\|_{\infty}\left|\partial_{x}\left(v_{f}-v_{a}\right)\right|+\left\|\partial_{x} v_{f}\right\|_{\infty}\left\|\partial_{v v}^{2} F\right\|_{\infty}\left|v_{f}-v_{a}\right| .
$$

The Lemmas 3.2 and 3.4 then yield

$\sup _{s \in \mathbb{R}}\left|\partial_{x}(f-g)(s, x, t)\right| \leq\left(\left\|\partial_{v} F\right\|_{\infty}\left(\Sigma^{\prime}+\frac{2}{\delta} \Sigma\right)+\left\|\partial_{x} v_{f}\right\|_{\infty}\left\|\partial_{v v}^{2} F\right\|_{\infty} \Sigma\right) \mathbf{1}_{(-R, R)}(x)$.

Estimate (49) follows from integrating on $\mathbb{R} \times\left(0, t^{\star}\right)$.

We aim to let $\delta, \Sigma$ and $\Sigma^{\prime}$ go to 0 , but we expect to select a good scaling so that the error contributions for $C_{1}, C_{2}$ and $C_{3}$ can be balanced. The first step consists 
of course in balancing the error contribution within each $C_{i}$. To do so, it appears from (45) and (49) that it is relevant to fix

$$
\Sigma^{\prime}=\frac{\Sigma}{\delta}
$$

We also propose to choose $\Sigma^{\prime \prime}$ of the same order as 1 , and for the sake of simplicity, we denote

$$
\Sigma^{\prime \prime}=1
$$

Because we investigate the limit $\delta, \Sigma, \Sigma^{\prime} \rightarrow 0$, this implies that $\Sigma$ is negligible w.r.t. $\Sigma^{\prime}=\Sigma / \delta$. As a consequence of the choices (50) and (51), we obtain for Lemmas 3.6, 3.7 and 3.8 that

$$
C_{1} \leq c\left(1+\frac{\Sigma}{\delta^{2}}\right), \quad C_{2} \leq c \Sigma, \quad \text { and } \quad C_{3} \leq c \frac{\Sigma}{\delta} .
$$

Now, balancing the contributions in $C_{1}$ suggests us to choose

$$
\delta=\Sigma^{1 / 2}
$$

so that (52) turns to

$$
C_{1} \leq c, \quad C_{2} \leq c \Sigma, \quad \text { and } \quad C_{3} \leq c \Sigma^{1 / 2},
$$

where $c$ denotes a generic quantity depending on the data and on $t^{\star}$ in an increasing way.

Theorem 3.9. Fix $\Sigma>0$, then with the choices (50) and (53) of the parameters $\delta$ and $\Sigma^{\prime}$, there exists $c$ depending only on $u_{0}, F, v_{f}, R$ and $T$ such that

$$
\left\|u_{a}-u_{f}\right\|_{\mathcal{C}\left([0, T] ; L^{1}(\mathbb{R})\right)} \leq c \Sigma^{1 / 2} .
$$

Proof. Let $t^{\star} \in[0, T]$, then taking (54) into account in (41) provides that

$$
\left\|u_{a}\left(\cdot, t^{\star}\right)-u_{f}\left(\cdot, t^{\star}\right)\right\|_{L^{1}(\mathbb{R})} \leq c\left(t^{\star}\right) \inf _{\epsilon>0}\left(\epsilon+\frac{\Sigma}{\epsilon}+\Sigma^{1 / 2}\right),
$$

where the dependence of $c$ w.r.t. $t^{\star}$ has been stressed. Therefore, since $c$ depends in an increasing way on $t^{\star}$, we deduce that

$$
\left\|u_{a}\left(\cdot, t^{\star}\right)-u_{f}\left(\cdot, t^{\star}\right)\right\|_{L^{1}(\mathbb{R})} \leq c(T) \inf _{\epsilon>0}\left(\epsilon+\frac{\Sigma}{\epsilon}+\Sigma^{1 / 2}\right) .
$$

The optimal choice for $\epsilon$ for minimizing the right hand-side is clearly $\epsilon=\Sigma^{1 / 2}$, yielding

$$
\left\|u_{a}\left(\cdot, t^{\star}\right)-u_{f}\left(\cdot, t^{\star}\right)\right\|_{L^{1}(\mathbb{R})} \leq c(T) \Sigma^{1 / 2} .
$$

Since both $u_{a}$ and $u_{f}$ belong to $\mathcal{C}\left([0, T] ; L_{\text {loc }}^{1}(\mathbb{R})\right.$ ) (see e.g. [6]) and since $u_{a}-u_{f}$ is compactly supported in space with support in $[-R, R]$, we obtain that $u_{a}-u_{f}$ belongs to $\mathcal{C}\left([0, T] ; L^{1}(\mathbb{R})\right)$ and that (55) holds. 


\section{Applichtion to A Model of transport With inertia}

In this section, we illustrate the dynamic model adaptation procedure on the simple example of a transport equation. Let $v_{\text {eq }} \in \mathcal{C}^{2}\left(\mathbb{R} \times \mathbb{R}_{+}\right)$be the material speed of the flow, that is supposed to be given. Then we are interested by the concentration $u$ of a chemical component convected by the flow. The coarse model consists in assuming that the speed of the chemical particles is exactly given by $v_{\text {eq. }}$. This yields the equation

$$
\left\{\begin{array}{l}
\partial_{t} u_{c}+\partial_{x}\left(v_{\mathrm{eq}} u_{c}\right)=0 \\
u_{c}(0)=u_{0}
\end{array}\right.
$$

But taking into account the inertia of the particles leads to considering that their speed does not coincide with the material speed of the flow, but is given by $v_{f}$ defined by the ODE

$$
\left\{\begin{array}{l}
\partial_{t} v_{f}=\frac{1}{\tau}\left(v_{\mathrm{eq}}-v_{f}\right), \\
v_{f}(\cdot, 0)=v_{0} .
\end{array}\right.
$$

The resulting concentration $u_{f}$ obeys the equation

$$
\left\{\begin{array}{l}
\partial_{t} u_{f}+\partial_{x}\left(v_{f} u_{f}\right)=0 \\
u_{f}(0)=u_{0}
\end{array}\right.
$$

where $v_{f}$ is given by (57).

As soon as the concentrations $u_{f}$ and $u_{c}$ remain uniformly bounded, the problem enters the frame proposed in Section 2. Therefore, we can apply the dynamic adaptation procedure described in Section 2.3 while controlling the error through the analysis carried out in Section 3.
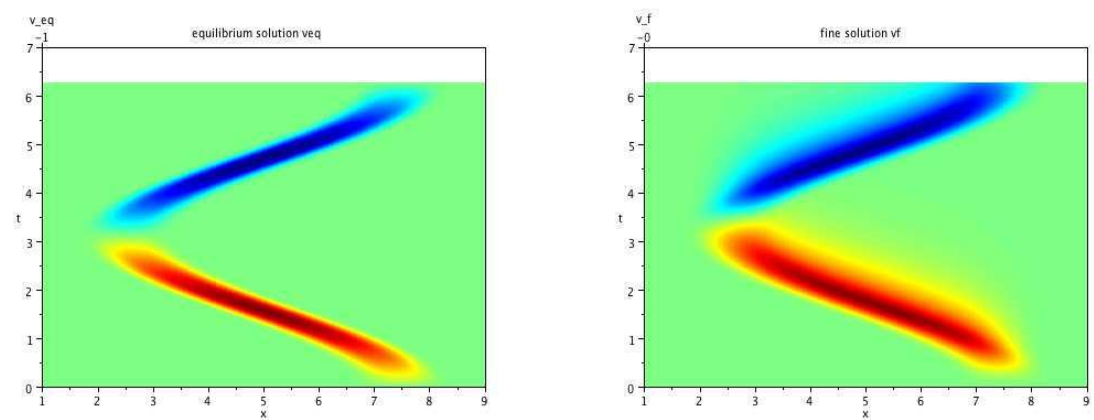

FIGURE 1. The equilibrium speed $v_{\text {eq }}$ (left) and the effective speed $v_{f}$ (right) plotted in the $(x, t)$-plan.

In order to illustrate our purpose, we have computed numerically the solutions $u_{c}, u_{f}$ and $u_{a}$ thanks to an explicit upwind finite volume scheme. The speed $v_{f}$, as well as the speed $v_{i}$ necessary to build $v_{a}$ are computed thanks to a fourth order Runge-Kutta scheme. 
We define the function $\nu$ by

$$
\nu(x)= \begin{cases}1 & \text { if } x \leq 0, \\ 1-8 x^{3}(1-x) & \text { if } x \in[0,1 / 2] \\ 1-\nu(1-x) & \text { if } x \geq 1 / 2\end{cases}
$$

then the equilibrium speed, plotted in $(x, t)$-plan on Figure 1, is given by

$$
v_{\text {eq }}(x, t)=\nu(|x-5-2.5 \cos (t)|)+0.3 \text {. }
$$

The relaxation parameter $\tau$ is set equal to 0.5 .

For the adaptation time step, we have set $\Delta t_{a}=\pi / 50$, and the numerical time step has been set to $\Delta t=\Delta t_{a} / 10$. Following the analysis carried out in Section 3 , we use $\Sigma$ as a parameter, and $\Sigma^{\prime}, \Sigma^{\prime \prime}$ and $\delta$ are fixed by

$$
\Sigma^{\prime}=\delta=\Sigma^{1 / 2}, \quad \Sigma^{\prime \prime}=1
$$

We define $u_{0}=\mathbf{1}_{x \leq 5}$, so that we can build the functions $u_{f}, u_{c}$ and $u_{a}$ for any choice of the parameter $\Sigma$.

We present now the results obtained for $\Sigma=0.1$. On Figures 2 and 3, we can see that all the solutions look similar. Nevertheless, it appears on Figure 4 that, as expected by our study, the adapted solution $u_{a}$ is much closer to the fine solution $u_{f}$ than $u_{c}$.
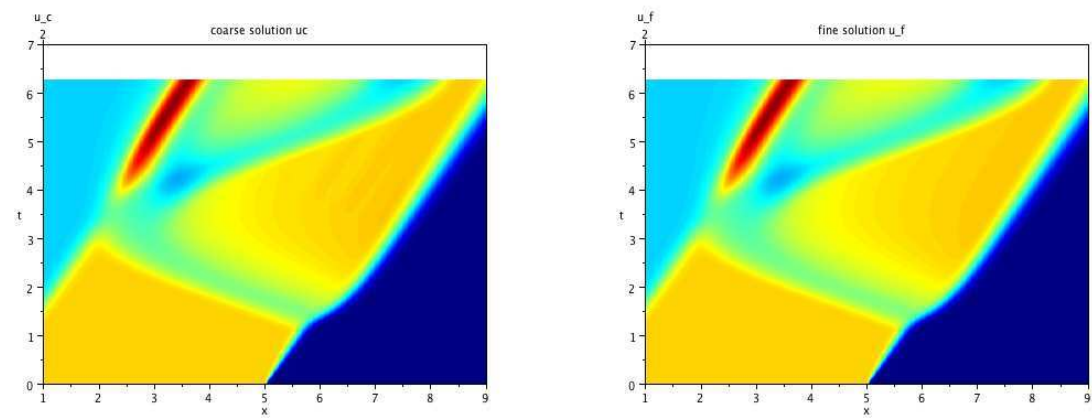

Figure 2. The concentration $u_{c}$ computed with the coarse model (56) (left) and the concentration $u_{f}$ computed with the fine model (58) (right) plotted in the $(x, t)$-plan.

We plot some results obtained for the lower value of $\Sigma=0.01$ on Figure 5 . As expected, the fine domain $\Omega_{f}$ in this case is bigger that in the case where $\Sigma=0.1$, and the error $\left|u_{a}-u_{f}\right|$ is smaller.

Finally, we present on Figure 6 the $L^{1}((1,9) \times(0,2 \pi))$-error between the fine solution $u_{f}$ and the adapted solution $u_{a}$. The saturation of the convergence if due to the numerical approximation of the solution. Indeed, refining the space and time discretization makes the saturation value decrease. 

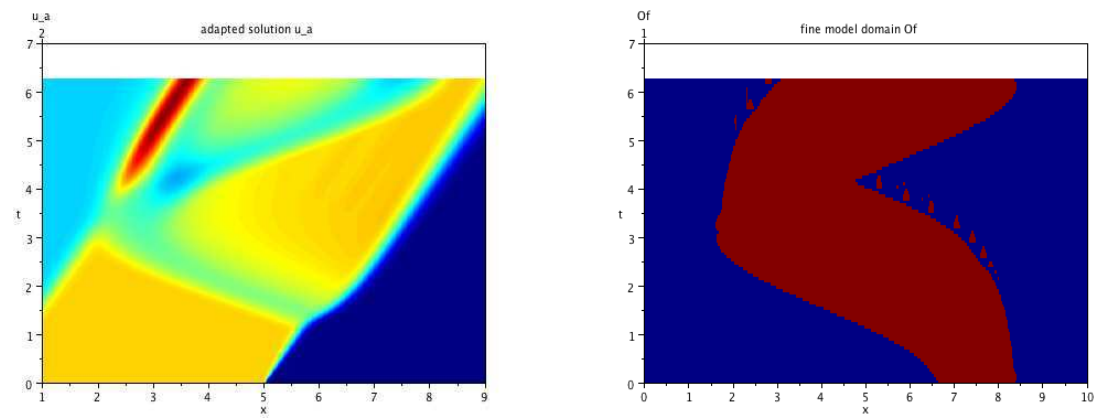

Figure 3. The concentration $u_{a}$ computed via the adapted model described in Section 2.3 (left) and the evolution of the domains $\Omega_{f}$ (red) and $\Omega_{c}$ (blue) w.r.t. time (right) plotted in the $(x, t)$-plan. $\Sigma=0.1$.
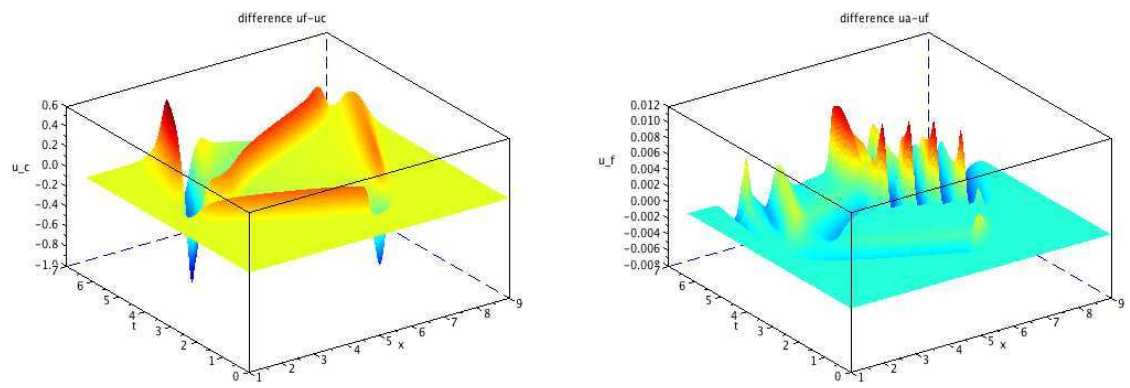

Figure 4. Plot in the $(x, t)$-plane of the difference between the fine and the coarse solutions $u_{c}-u_{f}$ (left), and the difference between the fine and the adapted solution $u_{a}-u_{f}$ (right, $\Sigma=0.1$ ). Notice the difference in the scales along the vertical axes.

\section{Conclusion}

We derive in this work an error estimate for a simple algorithm of dynamic model adaptation applied to nonlinear hyperbolic equations. In order to perform this analysis, we have to consider thick interfaces of coupling (the only error estimate with a thin coupling interface is available in the very particular case of [7]). Using Theorem 3.9, we are able to define the size $\delta$ of the smooth buffer which connects the fine model to the coarse model w.r.t. the parameters of the model and of the procedure. Note that we had to adapt the stability results obtained by LécureuxMercier in [23] in our context. We also provide some numerical results to illustrate the optimality of our result.

We only concentrate on a discrete-in-time procedure for the model adaptation. It would be interesting to include in the analysis the numerical error to obtain a full numerical procedure of model adaptation, as done for instance in [5]. The 

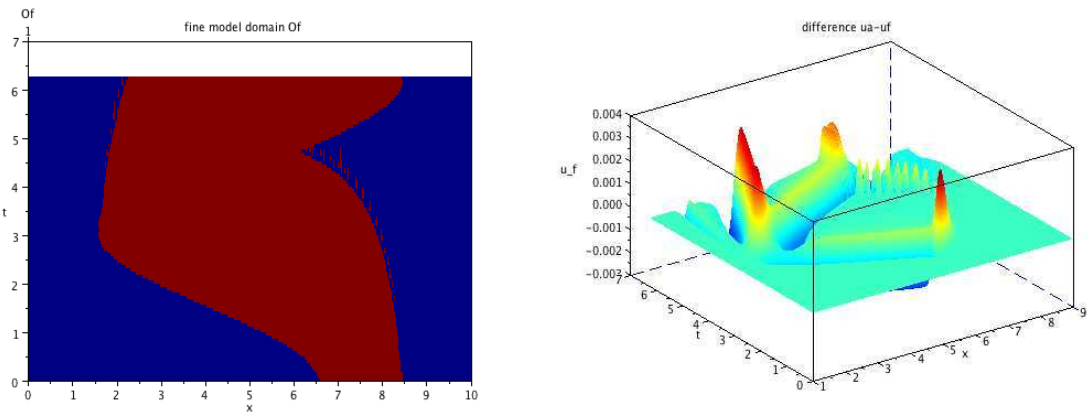

Figure 5. We plot in the $(x, t)$-plane the results of computations for $\Sigma=0.01$, i.e. the fine domain $\Omega_{f}(t)$ (in red, left), and the difference $u_{a}-u_{f}$ (right). For the left figure, check that the size of the fine domain grows when $\Sigma$ decreases. For the right figure, notice the difference in the scale along the vertical axes w.r.t. Figure 4.

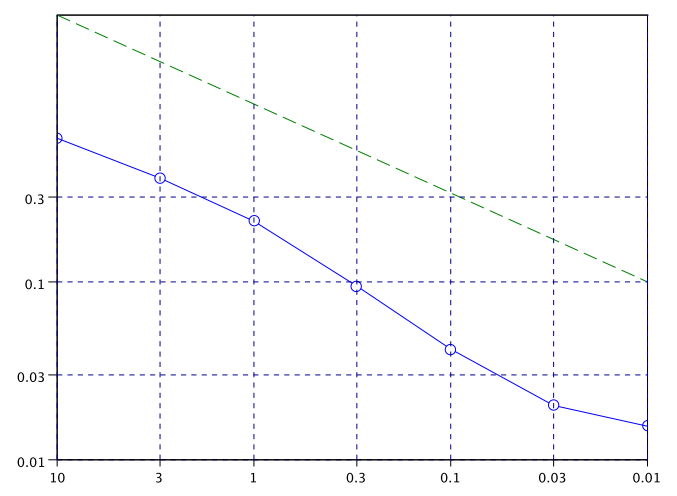

Figure 6. Numerical evaluation of $\left\|u_{a}-u_{f}\right\|_{L^{1}}$ as a function of $\Sigma$ (solid blue), linear function with slope $1 / 2$ (dashed green).

main tools can be found in the works of Chainais-Hillairet [8] and of Kröner and Ohlberger [20]. However, since our method is discrete w.r.t. time, the flux $f$ of the underlying scalar conservation law

$$
\partial_{t} u+\partial_{x} f(u, x, t)=0
$$

is discontinuous w.r.t. at each time step. As a consequence, we need to slightly refine the result of Chainais-Hillairet.

\section{Appendix A. Stability Results for scalar COnSERVATion LaWs}

In this appendix (precisely, in Theorem A.3), we state a new stability result for entropy solutions of scalar conservation law w.r.t. their flux functions. Despite all 
this section is written in the one-dimensional space dimension, it can be adapted to the multidimensional case without particular difficulty, excepted the heavy notations involved in the study.

The stability result presented in Theorem A.3 is an extension to the case of time and space dependent flux functions of a result presented in [3]. It relies on stability estimates proved in [23] (see also [12]) that are recalled in Theorem A.1.

A.1. Total variation estimates for Kružkov entropy solutions. We consider two functions

$$
f:\left\{\begin{array}{l}
\mathbb{R} \times \mathbb{R} \times \mathbb{R}_{+} \rightarrow \mathbb{R} \\
(s, x, t) \mapsto f(s ; x, t),
\end{array} \quad \text { and } \quad g:\left\{\begin{array}{l}
\mathbb{R} \times \mathbb{R} \times \mathbb{R}_{+} \rightarrow \mathbb{R} \\
(s, x, t) \mapsto f(s ; x, t),
\end{array}\right.\right.
$$

being continuous on $\mathbb{R} \times \mathbb{R} \times \mathbb{R}_{+}$, and having continuous derivatives $\partial_{s} f, \partial_{s} \partial_{x} f$ and $\partial_{x x}^{2} f$ (resp. $\partial_{s} g, \partial_{s} \partial_{x} g$ and $\partial_{x x}^{2} g$ ). We assume that $f(0 ; x, t)=g(0 ; x, t)$ for all $(x, t) \in \mathbb{R} \times \mathbb{R}_{+}$, and that

$$
\begin{aligned}
& L_{f}:=\sup _{(x, t) \in \mathbb{R} \times \mathbb{R}_{+}} \sup _{s_{1}, s_{2} \in \mathbb{R}}\left|\frac{f\left(s_{1} ; x, t\right)-f\left(s_{2} ; x, t\right)}{s_{1}-s_{2}}\right|<\infty, \\
& L_{g}:=\sup _{(x, t) \in \mathbb{R} \times \mathbb{R}_{+}} \sup _{s_{1}, s_{2} \in \mathbb{R}}\left|\frac{g\left(s_{1} ; x, t\right)-g\left(s_{2} ; x, t\right)}{s_{1}-s_{2}}\right|<\infty,
\end{aligned}
$$

that

$$
\begin{aligned}
\left\|\partial_{s} \partial_{x} f\right\|_{\infty} & :=\sup _{s \in \mathbb{R}} \sup _{(x, t) \in \mathbb{R} \times \mathbb{R}_{+}}\left|\partial_{s} \partial_{x} f(s ; x, t)\right|<\infty, \\
\left\|\partial_{s} \partial_{x} g\right\|_{\infty} & :=\sup _{s \in \mathbb{R}} \sup _{(x, t) \in \mathbb{R} \times \mathbb{R}_{+}}\left|\partial_{s} \partial_{x} g(s ; x, t)\right|<\infty,
\end{aligned}
$$

and that, for all $T>0$, for $\phi \in\{f, g\}$,

$$
(x, t) \mapsto\left\|\partial_{x x}^{2} \phi(\cdot ; x, t)\right\|_{\infty} \text { belongs to } L^{1}(\mathbb{R} \times(0, T)) \cap L^{\infty}\left(\mathbb{R} ; L^{1}(0, T)\right) .
$$

Let $u \in L^{\infty}\left(\mathbb{R} \times \mathbb{R}_{+}\right)$be the unique entropy solution of

$$
\left\{\begin{array}{l}
\partial_{t} u+\partial_{x} f(u ; x, t)=0 \quad \text { in } \mathbb{R} \times \mathbb{R}_{+}, \\
u(\cdot, 0)=u_{0} \in L^{\infty}(\mathbb{R}),
\end{array}\right.
$$

and let $v \in L^{\infty}\left(\mathbb{R} \times \mathbb{R}_{+}\right)$be the unique entropy solution of

$$
\left\{\begin{array}{l}
\partial_{t} v+\partial_{x} g(v ; x, t)=0 \quad \text { in } \mathbb{R} \times \mathbb{R}_{+}, \\
v(\cdot, 0)=v_{0} \in L^{\infty}(\mathbb{R}) .
\end{array}\right.
$$

Recall that $u$ is defined by: $\forall \kappa \in \mathbb{R}, \forall \psi \in \mathcal{C}_{c}^{\infty}\left(\mathbb{R} \times \mathbb{R}_{+}\right)$,

$$
\begin{aligned}
& \iint_{\mathbb{R} \times \mathbb{R}_{+}}|u-\kappa| \partial_{t} \psi d x d t+\int_{\mathbb{R}}\left|u_{0}-\kappa\right| \psi(\cdot, 0) d x \\
& \quad+\iint_{\mathbb{R}^{\prime} \mathbb{R}_{+}} \operatorname{sign}(u-\kappa)(f(u ; x, t)-f(\kappa ; x, t)) \partial_{x} \psi d x d t \\
& \quad-\iint_{\mathbb{R} \times \mathbb{R}_{+}} \operatorname{sign}(u-\kappa) \partial_{x} f(\kappa ; x, t) \psi d x d t \geq 0,
\end{aligned}
$$


while $v$ is defined by: $\forall \kappa \in \mathbb{R}, \forall \psi \in \mathcal{C}_{c}^{\infty}\left(\mathbb{R} \times \mathbb{R}_{+}\right)$,

$$
\begin{aligned}
& \iint_{\mathbb{R} \times \mathbb{R}_{+}}|v-\kappa| \partial_{t} \psi d x d t+\int_{\mathbb{R}}\left|v_{0}-\kappa\right| \psi(\cdot, 0) d x \\
& \quad+\iint_{\mathbb{R}^{\prime} \times \mathbb{R}_{+}} \operatorname{sign}(v-\kappa)(g(v ; x, t)-g(\kappa ; x, t)) \partial_{x} \psi d x d t \\
& \quad-\iint_{\mathbb{R}^{\prime} \times \mathbb{R}_{+}} \operatorname{sign}(v-\kappa) \partial_{x} g(\kappa ; x, t) \psi d x d t \geq 0 .
\end{aligned}
$$

Theorem A.1 ([6, 23]). The unique entropy solution $u$ to the problem (60) belongs to $\mathcal{C}\left(\mathbb{R}_{+}, L_{\text {loc }}^{1}(\mathbb{R})\right)$ and

$$
\lim _{t \rightarrow 0}\left\|u(\cdot, t)-u_{0}\right\|_{L^{1}(\mathbb{R})}=0 .
$$

Moreover, if $u_{0}$ belongs to $B V(\mathbb{R})$, then, for all $T>0, u(\cdot, T) \in B V(\mathbb{R})$, and there exists $c_{f}$ depending only on $f$ such that

$$
\operatorname{TV}(u(T)) \leq \operatorname{TV}\left(u_{0}\right) e^{c_{f} T}+\int_{0}^{T} e^{c_{f}(T-t)} \int_{\mathbb{R}}\left\|\partial_{x x}^{2} f(\cdot ; x, t)\right\|_{\infty} d x d t
$$

where

$$
\left\|\partial_{x x}^{2} f(\cdot ; x, t)\right\|_{\infty}:=\sup _{s \in \mathbb{R}}\left|\partial_{x x}^{2} f(s ; x, t)\right|
$$

and

$$
c_{f}=3\left\|\partial_{x} \partial_{s} f\right\|_{L^{\infty}\left(\mathbb{R} \times \mathbb{R} \times \mathbb{R}_{+}\right)} .
$$

In what follows, we will often have to deal with the quantity

$$
\int_{0}^{T} \operatorname{TV}(u(t)) d t=\operatorname{TV}\left(u_{0}\right) \frac{e^{c_{f} T}-1}{c_{f}}+\int_{0}^{T} \int_{0}^{t} e^{c_{f}(t-\tau)} \int_{\mathbb{R}}\left\|\partial_{x x}^{2} f(\cdot ; x, t)\right\|_{\infty} d x d \tau d t .
$$

We denote by

$$
\left\{\begin{array}{l}
C_{f, 0}(T)=\frac{e^{c_{f} T}-1}{c_{f}} \\
C_{f, 1}(T)=\int_{0}^{T} \int_{0}^{t} e^{c_{f}(t-\tau)} \int_{\mathbb{R}}\left\|\partial_{x x}^{2} f(\cdot ; x, t)\right\|_{\infty} d x d \tau d t
\end{array}\right.
$$

so that

$$
\int_{0}^{T} \operatorname{TV}(u(t)) d t=C_{f, 0}(T) \operatorname{TV}\left(u_{0}\right)+C_{f, 1}(T)
$$

Similarly, we have

$$
\int_{0}^{T} \operatorname{TV}(v(t)) d t=C_{g, 0}(T) \operatorname{TV}\left(v_{0}\right)+C_{g, 1}(T)
$$

where the quantities $C_{g, 0}$ and $C_{g, 1}$ are obtained from $C_{f, 0}$ and $C_{f, 1}$ by replacing the flux function $f$ by the flux function $g$.

A.2. Stability w.r.t. the flux functions. We first state the following technical lemma. 
Lemma A.2. For all $\kappa \in \mathbb{R}$, there exists $A_{\kappa}, B_{\kappa} \in L^{\infty}\left(\mathbb{R} \times \mathbb{R}_{+}\right)$such that

$$
\begin{aligned}
& \iint_{\mathbb{R} \times \mathbb{R}_{+}}|v-\kappa| \partial_{t} \psi d x d t+\int_{\mathbb{R}}\left|v_{0}-\kappa\right| \psi(\cdot, 0) d x \\
& \quad+\iint_{\mathbb{R}^{\prime} \mathbb{R}_{+}} \operatorname{sign}(v-\kappa)(f(v ; x, t)-f(\kappa ; x, t)) \partial_{x} \psi d x d t \\
& \quad-\iint_{\mathbb{R}^{\prime} \mathbb{R}_{+}} \operatorname{sign}(v-\kappa) \partial_{x} f(\kappa ; x, t) \psi d x d t \\
& \geq \iint_{\mathbb{R}^{\prime} \times \mathbb{R}_{+}} A_{\kappa} \partial_{x} \psi d x d t+\iint_{\mathbb{R}^{\prime} \times \mathbb{R}_{+}} B_{\kappa} \psi d x d t .
\end{aligned}
$$

Moreover, for all $\kappa \in \mathbb{R}$,

$$
\left|A_{\kappa}(x, t)\right| \leq 2\|(f-g)(\cdot, x, t)\|_{\infty}, \quad \forall(x, t) \in \mathbb{R} \times \mathbb{R}_{+},
$$

while

$$
\left|B_{\kappa}(x, t)\right| \leq\left\|\partial_{x}(f-g)(\cdot ; x, t)\right\|_{\infty}, \quad \forall(x, t) \in \mathbb{R} \times \mathbb{R}_{+} .
$$

Proof. First of all, note that, thanks to (63), the function $v$ satisfies : $\forall \kappa \in \mathbb{R}$, $\forall \psi \in \mathcal{C}_{c}^{\infty}\left(\mathbb{R} \times \mathbb{R}_{+}\right)$,

$$
\begin{aligned}
& \iint_{\mathbb{R} \times \mathbb{R}_{+}}|v-\kappa| \partial_{t} \psi d x d t+\int_{\mathbb{R}}\left|v_{0}-\kappa\right| \psi(\cdot, 0) d x \\
& +\iint_{\mathbb{R}^{\prime} \times \mathbb{R}_{+}} \operatorname{sign}(v-\kappa)(f(v ; x, t)-f(\kappa ; x, t)) \partial_{x} \psi d x d t \\
& -\iint_{\mathbb{R} \times \mathbb{R}_{+}} \operatorname{sign}(v-\kappa) \partial_{x} f(\kappa ; x, t) \psi d x d t \\
& \geq \iint_{\mathbb{R} \times \mathbb{R}_{+}} A_{\kappa} \partial_{x} \psi d x d t+\iint_{\mathbb{R} \times \mathbb{R}_{+}} B_{\kappa} \psi d x d t,
\end{aligned}
$$

where

$$
\begin{aligned}
& A_{\kappa}(x, t)=\operatorname{sign}(v(x, t)-\kappa)[(f-g)(v(x, t) ; x, t)-(f-g)(\kappa ; x, t)], \\
& B_{\kappa}(x, t)=\operatorname{sign}(v(x, t)-\kappa) \partial_{x}(g-f)(\kappa ; x, t) .
\end{aligned}
$$

Clearly, the relations (68) and (69) hold for all $\kappa \in \mathbb{R}$.

We now give a stability result which is an adaptation of Theorem 3.1 of [3] in the case of time-space depending flux functions. For $x_{0} \in \mathbb{R}$ and $R>0$, we denote by

$$
B\left(x_{0}, R\right)=\left\{x \in \mathbb{R}|| x-x_{0} \mid<\mathbb{R}\right\},
$$

and, for $t \in[0, t)$ and $\epsilon>0$,

$$
B_{t}=\left\{x \in \mathbb{R}|| x \mid<R+L_{f}(T-t)\right\}, \quad B_{t}^{\epsilon}=\left\{y \in \mathbb{R}|| y \mid<R+L_{f}(T-t)+\epsilon\right\},
$$

Theorem A.3. Let $x_{0} \in \mathbb{R}$, and let $u_{0} \in \mathrm{BV}(\mathbb{R})$, then, for all $T>0$ and for all $R>0$,

$$
\|u(\cdot, T)-v(\cdot, T)\|_{L^{1}\left(B\left(x_{0}, R\right)\right)} \leq\left\|u_{0}-v_{0}\right\|_{L^{1}\left(B\left(x_{0}, R+L_{f} T\right)\right)}+\inf _{\epsilon>0}\left(\epsilon C_{1}^{\epsilon}+\frac{C_{2}^{\epsilon}}{\epsilon}+C_{3}^{\epsilon}\right),
$$


where

$$
\begin{aligned}
C_{1}^{\epsilon}:= & \operatorname{TV}\left(v_{0}\right)\left(1+e^{c_{g} T}+2\left\|\partial_{s} \partial_{x} f\right\|_{\infty} C_{g, 0}(T)\right) \\
& +2\left\|\partial_{s} \partial_{x} f\right\|_{\infty} C_{g, 1}(T)+3 \int_{0}^{T} \sup _{s \in \mathbb{R}}\left\|\partial_{x x}^{2} f(s, \cdot, t)\right\|_{L^{\infty}\left(B_{t}^{\epsilon}\right)}, \\
C_{2}^{\epsilon}:= & 2 \int_{0}^{T} \int_{y \in B_{t}^{\epsilon}}\|(f-g)(\cdot ; y, t)\|_{\infty} d y d t \\
C_{3}^{\epsilon}:= & \int_{0}^{T} \int_{y \in B_{t}^{\epsilon}}\left\|\partial_{y}(f-g)(\cdot ; y, t)\right\|_{\infty} d y d t .
\end{aligned}
$$

Proof. For the sake of simplicity, we only perform the proof for $x_{0}=0$, but clearly, adapting it to any $x_{0} \in \mathbb{R}$ does not provide any additional difficulty. We follow the idea of Kružkov [21], Kuznetsov [22] and Bouchut and Perthame [3], and carry out a proof based on the doubling variable technique. Let $\xi: \mathbb{R} \times \mathbb{R}_{+} \times \mathbb{R} \times \mathbb{R}_{+} \rightarrow \mathbb{R}_{+}$ be a smooth and compactly supported function. Then it follows from (62) and (70) that

$$
\begin{aligned}
& \iint_{\mathbb{R} \times \mathbb{R}_{+}} \iint_{\mathbb{R} \times \mathbb{R}_{+}}|u(x, t)-\kappa| \partial_{t} \xi(x, t, y, s) d x d t d y d s \\
& \quad+\iint_{\mathbb{R} \times \mathbb{R}_{+}} \int_{\mathbb{R}}\left|u_{0}(x)-\kappa\right| \xi(x, 0, y, s) d x d y d s \\
& \quad+\iint_{\mathbb{R} \times \mathbb{R}_{+}} \iint_{\mathbb{R} \times \mathbb{R}_{+}}\left\{\begin{array}{r}
\operatorname{sign}(u(x, t)-\kappa) \times \\
(f(u(x, t) ; x, t)-f(\kappa ; x, t)) \partial_{x} \xi(x, t, y, s)
\end{array}\right\} d x d t d y d s \\
& \quad-\iint_{\mathbb{R}^{\prime} \times \mathbb{R}_{+}} \iint_{\mathbb{R} \times \mathbb{R}_{+}} \operatorname{sign}(u(x, t)-\kappa) \partial_{x} f(\kappa ; x, t) \xi(x, t, y, s) d x d t d y d s \geq 0, \quad(70)
\end{aligned}
$$

while

$$
\begin{aligned}
\iint_{\mathbb{R} \times \mathbb{R}_{+}} & \iint_{\mathbb{R} \times \mathbb{R}_{+}}|v(y, s)-\kappa| \partial_{s} \xi(x, t, y, s) d x d t d y d s \\
& +\iiint_{\mathbb{R}_{\mathbb{R}} \mathbb{R}_{+}}\left|v_{0}(y)-\kappa\right| \xi(x, t, y, 0) d x d t d y \\
& +\iint_{\mathbb{R} \times \mathbb{R}_{+}} \iint_{\mathbb{R} \times \mathbb{R}_{+}}\left\{\begin{array}{r}
\operatorname{sign}(v(y, s)-\kappa) \times \\
(f(v(y, s) ; y, s)-f(\kappa ; y, s)) \partial_{y} \xi(x, t, y, s)
\end{array}\right\} d x d t d y d s \\
& -\iint_{\mathbb{R} \times \mathbb{R}_{+}} \iint_{\mathbb{R} \times \mathbb{R}_{+}} \operatorname{sign}(v(y, s)-\kappa) \partial_{y} f(\kappa ; y, s) \xi(x, t, y, s) d x d t d y d s \\
\geq & \iint_{\mathbb{R} \times \mathbb{R}_{+}} \iint_{\mathbb{R} \times \mathbb{R}_{+}}\left[B_{\kappa}(y, s) \xi(x, t, y, s)+A_{\kappa}(y, s) \partial_{y} \xi(x, t, y, s)\right] d x d t d y d s . \text { (71) }
\end{aligned}
$$

Let $\rho, \tilde{\rho} \in \mathcal{C}_{c}^{\infty}\left(\mathbb{R} ; \mathbb{R}_{+}\right)$such that $\int_{\mathbb{R}} \rho(s) d s=\int_{\mathbb{R}} \tilde{\rho}(s) d s=1$ and $\operatorname{supp} \rho \subset[-1,1]$, $\operatorname{supp} \tilde{\rho} \subset[-1,0]$. We moreover assume that

$$
\rho(0)=1 \text {, and } s \rho^{\prime}(s) \leq 0, \quad \forall s \in \mathbb{R} .
$$

Then, for $\epsilon, \delta>0$, we denote by

$$
\rho_{\epsilon}(s)=\frac{1}{\epsilon} \rho\left(\frac{s}{\epsilon}\right), \quad \tilde{\rho}_{\delta}(s)=\frac{1}{\delta} \tilde{\rho}\left(\frac{s}{\delta}\right),
$$


so that $\operatorname{supp} \rho_{\epsilon} \subset[-\epsilon, \epsilon], \operatorname{supp} \tilde{\rho}_{\delta} \subset[-\delta, 0]$ and $\int_{\mathbb{R}} \rho_{\epsilon}(s) d s=\int_{\mathbb{R}} \tilde{\rho}_{\delta}(s) d s=1$. As a consequence of (72), one has also

$$
-s \rho_{\epsilon}^{\prime}(s) \geq 0 \quad \text { and } \quad-\int_{\mathbb{R}} s \rho_{\epsilon}^{\prime}(s) d s=1,
$$

ensuring that $s \mapsto-s \rho_{\epsilon}^{\prime}(s)$ is also an approximation of the unit.

Let $\psi \in \mathcal{C}_{c}^{\infty}(\mathbb{R} \times[0, T))$ (for some $T>0$ ), then, choosing

$$
\xi(x, t, y, s)=\psi(x, t) \rho_{\epsilon}(x-y) \tilde{\rho}_{\delta}(t-s)
$$

yields

$$
\begin{aligned}
\partial_{y} \xi(x, t, y, s) & =-\psi(x, t) \rho_{\epsilon}^{\prime}(x-y) \tilde{\rho}_{\delta}(t-s) \\
\partial_{t} \xi(x, t, y, s)+\partial_{s} \xi(x, t, y, s) & =\partial_{t} \psi(x, t) \rho_{\epsilon}(x-y) \tilde{\rho}_{\delta}(t-s) \\
\partial_{x} \xi(x, t, y, s)+\partial_{y} \xi(x, t, y, s) & =\partial_{x} \psi(x, t) \rho_{\epsilon}(x-y) \tilde{\rho}_{\delta}(t-s) .
\end{aligned}
$$

Choosing $\kappa=v(y, s)$ in (70) and $\kappa=u(x, t)$ in (71), then summing (recall that $\tilde{\rho}_{\delta}(s)=0$ if $\left.s \leq 0\right)$ provides

$$
T_{1}^{\epsilon, \delta}+T_{2}^{\epsilon, \delta}+T_{3}^{\epsilon, \delta}+T_{4}^{\epsilon, \delta} \geq T_{5}^{\epsilon, \delta}+T_{6}^{\epsilon, \delta}
$$

where

$$
\begin{aligned}
& T_{1}^{\epsilon, \delta}=\iint_{\mathbb{R}^{\prime} \mathbb{R}_{+}} \iint_{\mathbb{R}_{\mathbb{R}} \times \mathbb{R}_{+}}|u(x, t)-v(y, s)| \partial_{t} \psi(x, t) \rho_{\epsilon}(x-y) \tilde{\rho}_{\delta}(t-s) d x d t d y d s, \\
& T_{2}^{\epsilon, \delta}=\iint_{\mathbb{R} \times \mathbb{R}_{+}} \int_{\mathbb{R}}\left|u_{0}(x)-v(y, s)\right| \psi(x, 0) \rho_{\epsilon}(x-y) \tilde{\rho}_{\delta}(-s) d x d y d s, \\
& T_{3}^{\epsilon, \delta}=\iint_{\mathbb{R} \times \mathbb{R}_{+}} \iint_{\mathbb{R} \times \mathbb{R}_{+}}\left\{\begin{array}{r}
\operatorname{sign}(u(x, t)-v(y, s)) \times \\
(f(u(x, t) ; x, t)-f(v(y, s) ; x, t)) \\
\partial_{x} \psi(x, t) \rho_{\epsilon}(x-y) \tilde{\rho}_{\delta}(t-s)
\end{array}\right\} d x d t d y d s \\
& T_{4}^{\epsilon, \delta}=\iint_{\mathbb{R}^{\prime} \mathbb{R}_{+}} \iint_{\mathbb{R} \times \mathbb{R}_{+}}\left\{\begin{array}{r}
\operatorname{sign}(u(x, t)-v(y, s)) \times \\
{\left[(f(v(y, s) ; y, s)-f(v(y, s) ; x, t)) \rho_{\epsilon}^{\prime}(x-y)\right.} \\
-\partial_{x} f(v(y, s) ; x, t) \rho_{\epsilon}(x-y) \\
-(f(u(x, t) ; y, s)-f(u(x, t) ; x ; t)) \rho_{\epsilon}^{\prime}(x-y) \\
\left.+\partial_{y} f(u(x, t) ; y, s) \rho_{\epsilon}(x-y)\right] \times \\
\tilde{\rho}_{\delta}(t-s) \psi(x, t)
\end{array}\right\} d x d t d y d s, \\
& T_{5}^{\epsilon, \delta}=-\iint_{\mathbb{R} \times \mathbb{R}_{+}} \iint_{\mathbb{R}_{\mathbb{R}_{+}}} A_{u(x, t)}(y, s) \psi(x, t) \rho_{\epsilon}^{\prime}(x-y) \tilde{\rho}_{\delta}(t-s) d x d t d y d s, \\
& T_{6}^{\epsilon, \delta}=\iint_{\mathbb{R} \times \mathbb{R}_{+}} \iint_{\mathbb{R} \times \mathbb{R}_{+}} B_{u(x, t)}(y, s) \psi(x, t) \rho_{\epsilon}(x-y) \tilde{\rho}_{\delta}(t-s) d x d t d y d s .
\end{aligned}
$$

We can directly let $\delta$ tend to 0 in (74). Using the continuity in mean theorem and (64), this yields that

$$
T_{1}^{\epsilon}+T_{2}^{\epsilon}+T_{3}^{\epsilon}+T_{4}^{\epsilon} \geq T_{5}^{\epsilon}+T_{6}^{\epsilon}
$$


where

$$
\begin{aligned}
T_{1}^{\epsilon}= & \int_{\mathbb{R}} \iint_{\mathbb{R} \times \mathbb{R}_{+}}|u(x, t)-v(y, t)| \partial_{t} \psi(x, t) \rho_{\epsilon}(x-y) d x d t d y, \\
T_{2}^{\epsilon}= & \int_{\mathbb{R}} \int_{\mathbb{R}}\left|u_{0}(x)-v_{0}(y)\right| \psi(x, 0) \rho_{\epsilon}(x-y) d x d y, \\
T_{3}^{\epsilon}= & \int_{\mathbb{R}} \iint_{\mathbb{R} \times \mathbb{R}_{+}}\left\{\begin{array}{r}
\operatorname{sign}(u(x, t)-v(y, t)) \times \\
(f(u(x, t) ; x, t)-f(v(y, t) ; x, t)) \\
\partial_{x} \psi(x, t) \rho_{\epsilon}(x-y)
\end{array}\right\} d x d t d y, \operatorname{sign}(u(x, t)-v(y, t)) \times \\
T_{4}^{\epsilon}= & \int_{\mathbb{R}} \iint_{\mathbb{R} \times \mathbb{R}_{+}}\left\{\begin{array}{r}
+f(u(x, t) ; x, t)-f(u(x, t) ; y ; t) \rho_{\epsilon}^{\prime}(x-y) \\
\left.+\partial_{y} f(u(x, t) ; y, t) \rho_{\epsilon}(x-y)\right] \psi(x, t)
\end{array}\right\} d x d t d y, \\
T_{5}^{\epsilon}= & -\int_{\mathbb{R}} \iint_{\mathbb{R} \times \mathbb{R}_{+}} A_{u(x, t)}(y, t) \psi(x, t) \rho_{\epsilon}^{\prime}(x-y) d x d t d y, \\
T_{6}^{\epsilon}= & \int_{\mathbb{R}} \iint_{\mathbb{R} \times \mathbb{R}_{+}} B_{u(x, t)}(y, t) \psi(x, t) \rho_{\epsilon}(x-y) d x d t d y .
\end{aligned}
$$

Contrarily to the study performed by Kružkov, we cannot let the parameter $\epsilon$ tend to 0 , because of the presence of $\rho_{\epsilon}^{\prime}$ in $T_{6}^{\epsilon}$. The goal is now to derive $\epsilon$-dependent estimates from (75), as it was already the case in [22].

Define the functions $Y_{\theta}: s \mapsto \min (1 ; \max (0 ; 1-s / \theta))$ and

$$
\phi_{\theta}(x, t)=Y_{\theta}\left(|x|-R-L_{f}(T-t)\right),
$$

then, for a function $\chi \geq 0$ with compact support in $[0, T)$ to be specified latter, setting $\psi(x, t)=\phi_{\theta}(x, t) \chi(t)$ yields that

$$
\begin{aligned}
& |u(x, t)-v(y, t)| \phi_{\theta}(x, t) \chi^{\prime}(t) \\
& \geq|u(x, t)-v(y, t)| \partial_{t} \psi(x, t) \\
& \quad+\operatorname{sign}(u(x, t)-v(y, t))(f(u(x, t) ; x, t)-f(v(y, t) ; x, t)) \partial_{x} \psi(x, t) .
\end{aligned}
$$

Therefore,

$$
T_{1}^{\epsilon}+T_{3}^{\epsilon} \leq \int_{\mathbb{R}} \iint_{\mathbb{R} \times \mathbb{R}_{+}}|u(x, t)-v(y, t)| \phi_{\theta}(x, t) \chi^{\prime}(t) \rho_{\epsilon}(x-y) d x d y d t .
$$

For this choice of test function $\psi$, letting $\theta$ tend to 0 and recalling that

$$
B_{t}=\left\{x \in \mathbb{R}|| x \mid<R+L_{f}(T-t)\right\}, \quad B_{t}^{\epsilon}=\left\{y \in \mathbb{R}|| y \mid<R+L_{f}(T-t)+\epsilon\right\}
$$

taking (76) into account in the relation (75) provides

$$
D_{1}^{\epsilon}+D_{2}^{\epsilon}+D_{3}^{\epsilon} \leq D_{4}^{\epsilon}+D_{5}^{\epsilon},
$$


where

$$
\begin{aligned}
& D_{1}^{\epsilon}=\int_{0}^{T} \int_{y \in B_{t}^{\epsilon}} \int_{x \in B_{t}}|u(x, t)-v(y, t)| \chi^{\prime}(t) \rho_{\epsilon}(x-y) d x d y d t \\
& D_{2}^{\epsilon}=\int_{y \in B_{0}^{\epsilon}} \int_{x \in B_{0}}\left|u_{0}(x)-v_{0}(y)\right| \chi(0) \rho_{\epsilon}(x-y) d x d y \\
& D_{3}^{\epsilon}=\int_{0}^{T} \int_{y \in B_{t}^{\epsilon}} \int_{x \in B_{t}}\left\{\begin{array}{r}
\operatorname{sign}(u(x, t)-v(y, t)) \times \\
+f(u(x, t) ; x, t)-f(u(x, t) ; y ; t) \rho_{\epsilon}^{\prime}(x-y) \\
\left.+\partial_{y} f(u(x, t) ; y, t) \rho_{\epsilon}(x-y)\right] \chi(t)
\end{array}\right) \\
& D_{4}^{\epsilon}=\int_{0}^{T} \int_{y \in B_{t}^{\epsilon}} \int_{x \in B_{t}} A_{u(x, t)}(y, t) \rho_{\epsilon}^{\prime}(x-y) \chi(t) d x d y d t \\
& D_{5}^{\epsilon}=\int_{0}^{T} \int_{y \in B_{t}^{\epsilon}} \int_{x \in B_{t}} B_{u(x, t)}(y, s) \rho_{\epsilon}(x-y) \chi(t) d x d y d t .
\end{aligned}
$$

From the triangular inequality, one has

$$
D_{2}^{\epsilon} \leq \int_{x \in B_{0}}\left|u_{0}(x)-v_{0}(x)\right| \chi(0) d x+\int_{B_{0}^{\epsilon}} \int_{B_{0}}\left|v_{0}(x)-v_{0}(y)\right| \chi(0) \rho_{\epsilon}(x-y) d x d y .
$$

Since $v_{0}$ belongs to $\mathrm{BV}(\mathbb{R})$, then

$$
\int_{B_{0}^{\epsilon}} \int_{B_{0}}\left|v_{0}(x)-v_{0}(y)\right| \chi(0) \rho_{\epsilon}(x-y) d x d y \leq \epsilon \operatorname{TV}\left(v_{0}\right) \chi(0),
$$

thus one has

$$
D_{2}^{\epsilon} \leq \int_{x \in B_{0}}\left|u_{0}(x)-v_{0}(x)\right| \chi(0) d x+\epsilon \operatorname{TV}\left(v_{0}\right) \chi(0) .
$$

Similarly, the triangular inequality yields that

$$
\begin{aligned}
D_{1}^{\epsilon} \leq & \int_{0}^{T} \int_{x \in B_{t}}|u(x, t)-v(x, t)| \chi^{\prime}(t) d x d t \\
& +\int_{0}^{T}\left|\chi^{\prime}(t)\right| \int_{y \in B_{t}^{\epsilon}} \int_{x \in B_{t}}|v(x, t)-v(y, t)| \rho_{\epsilon}(x-y) d x d y d t .
\end{aligned}
$$

Since

$$
\int_{y \in B_{t}^{\epsilon}} \int_{x \in B_{t}}|v(x, t)-v(y, t)| \rho_{\epsilon}(x-y) d x d y \leq \operatorname{TV}(v(\cdot, t)) \epsilon,
$$

then it follows from Theorem A.1 that for all $t \in[0, T]$,

$$
\operatorname{TV}(v(\cdot, t)) \leq \operatorname{TV}\left(v_{0}\right) e^{c_{g} T}+\int_{0}^{T} e^{c_{g}(T-\tau)} \int_{\mathbb{R}}\left\|\partial_{x x}^{2} g(\cdot, x, t)\right\|_{\infty} d x d t:=C_{\mathrm{BV}},
$$

where $c_{g}=3\left\|\partial_{x} \partial_{s} g\right\|_{\infty}$. As a consequence,

$$
D_{1}^{\epsilon} \leq \int_{0}^{T} \int_{x \in B_{t}}|u(x, t)-v(x, t)| \chi^{\prime}(t) d x d t+\epsilon C_{\mathrm{BV}}\left\|\chi^{\prime}\right\|_{L^{1}} .
$$

Since $x \mapsto f(s ; x, t)$ belongs to $\mathcal{C}^{2}(\mathbb{R}, \mathbb{R})$, one has, for all $(x, y) \in B_{t} \times B_{t}^{\epsilon}$, that

$$
f(v(y, t) ; y, t)-f(v(y, t) ; x, t)=\partial_{x} f(v(y, t) ; x, t)(y-x)+(y-x)^{2} \mu(x, y, t),
$$


for some $\mu(x, y, t) \in \mathbb{R}$ with

$$
|\mu(x, y, t)| \leq \frac{1}{2} \sup _{s \in \mathbb{R}}\left\|\partial_{x x}^{2} f(s ; \cdot, t)\right\|_{L^{\infty}\left(B_{t}^{\epsilon}\right)} .
$$

Therefore,

$$
\begin{aligned}
& (f(v(y, t) ; y, t)-f(v(y, t) ; x, t)) \rho_{\epsilon}^{\prime}(x-y)-\partial_{x} f(v(y, t) ; x, t) \rho_{\epsilon}(x-y) \\
& \quad=\partial_{x} f(v(y, t) ; x, t) \partial_{x}\left((y-x) \rho_{\epsilon}(x-y)\right)+(x-y)^{2} \rho_{\epsilon}^{\prime}(x-y) \mu(x, y, t),
\end{aligned}
$$

and similarly,

$$
\begin{aligned}
& (f(u(x, t) ; x, t)-f(u(x, t) ; y, t)) \rho_{\epsilon}^{\prime}(x-y)+\partial_{y} f(u(x, t) ; y, t) \rho_{\epsilon}(x-y) \\
& \quad=\partial_{y} f(u(x, t) ; y, t) \partial_{y}\left((x-y) \rho_{\epsilon}(x-y)\right)-(x-y)^{2} \rho_{\epsilon}^{\prime}(x-y) \nu(x, y, t) \\
& \quad=-\partial_{y} f(u(x, t) ; y, t) \partial_{x}\left((x-y) \rho_{\epsilon}(x-y)\right)-(x-y)^{2} \rho_{\epsilon}^{\prime}(x-y) \nu(x, y, t),
\end{aligned}
$$

for some $\nu(x, y, t) \in \mathbb{R}$ fulfilling

$$
|\nu(x, y, t)| \leq \frac{1}{2} \sup _{s \in \mathbb{R}}\left\|\partial_{x x}^{2} f(s ; \cdot, t)\right\|_{L^{\infty}\left(B_{t}^{\epsilon}\right)} .
$$

It follows from (59) that $\mu$ and $\nu$ belong to $L^{1}(\mathbb{R} \times \mathbb{R} \times(0, T))$. Denoting by

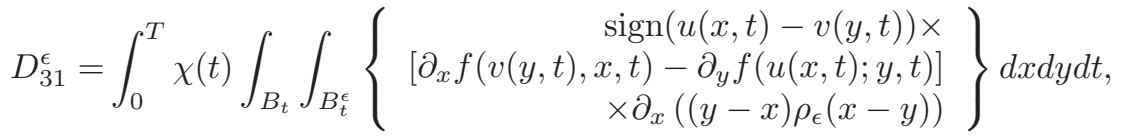

$$
\begin{aligned}
& D_{32}^{\epsilon}=\int_{0}^{T} \chi(t) \int_{B_{t}} \int_{B_{t}^{\epsilon}}\left\{\begin{array}{r}
\operatorname{sign}(u(x, t)-v(y, t)) \times \\
(\mu(x, y, t)+\nu(x, y, t))(x-y)^{2} \rho_{\epsilon}^{\prime}(x-y)
\end{array}\right\} d x d y d t,
\end{aligned}
$$

we have

$$
D_{3}^{\epsilon}=D_{31}^{\epsilon}+D_{32}^{\epsilon} \text {. }
$$

Clearly, for all $x \in \mathbb{R}$, the function $y \mapsto(x-y)^{2} \rho_{\epsilon}^{\prime}(x-y)$ is compactly supported in $[x-\epsilon, x+\epsilon]$ and

$$
\left|(x-y)^{2} \rho_{\epsilon}^{\prime}(x-y)\right| \leq \epsilon(y-x) \rho_{\epsilon}^{\prime}(x-y) .
$$

Using (72), (80) and (82), this ensures that

$$
\left|D_{32}^{\epsilon}\right| \leq \epsilon \int_{0}^{T} \chi(t) \sup _{s \in \mathbb{R}}\left\|\partial_{x x}^{2} f(s, \cdot, t)\right\|_{L^{\infty}\left(B_{t}^{\epsilon}\right)}\left(\int_{B_{t}} d x\right) d t
$$

Focus now on $D_{31}^{\epsilon}$, that we rewrite under the form

$$
D_{31}^{\epsilon}=\int_{0}^{T} \chi(t) \int_{x \in B_{t}} \int_{y \in B_{t}^{\epsilon}}[A(x, y, t)+B(x, y, t)] \partial_{x}\left[(y-x) \rho_{\epsilon}(x-y)\right] d x d y d t
$$

where

$$
\begin{aligned}
& A(x, y, t)=\operatorname{sign}(u(x, t)-v(y, t))\left(\tilde{\partial}_{x} f(v(y, t) ; x, t)-\tilde{\partial}_{x} f(u(x, t) ; x, t)\right), \\
& B(x, y, t)=\operatorname{sign}(u(x, t)-v(y, t))\left(\tilde{\partial}_{x} f(u(x, t) ; x, t)-\partial_{y} f(u(x, t) ; y, t)\right),
\end{aligned}
$$

where we have introduced the notation

$$
\tilde{\partial}_{x} f(u(x, t) ; x, t)=\lim _{h \rightarrow 0} \frac{f(u(x, t) ; x+h, t)-f(u(x, t) ; x, t)}{h} .
$$


Thanks to (72), one has

$$
\int_{\mathbb{R}} \partial_{x}\left[(y-x) \rho_{\epsilon}(x-y)\right] d y=0, \quad \forall x \in \mathbb{R}
$$

This implies that, for $\Upsilon \in\{A, B\}$,

$$
\begin{aligned}
\int_{0}^{T} \chi(t) \int_{x \in B_{t}} \int_{y \in B_{t}^{\epsilon}} \Upsilon(x, y, t) \partial_{x}\left[(y-x) \rho_{\epsilon}(x-y)\right] d y d x d t \\
=\int_{0}^{T} \chi(t) \int_{x \in B_{t}} \int_{y \in B_{t}^{\epsilon}}[\Upsilon(x, y, t)-\Upsilon(x, x, t)] \partial_{x}\left[(y-x) \rho_{\epsilon}(x-y)\right] d y d x d t \\
\leq \int_{0}^{T} \chi(t) \int_{x \in B_{t}} \int_{y \in B_{t}^{\epsilon}}|\Upsilon(x, y, t)-\Upsilon(x, x, t)|(y-x) \rho_{\epsilon}^{\prime}(x-y) d y d x d t \\
\quad+\int_{0}^{T} \chi(t) \int_{x \in B_{t}} \int_{y \in B_{t}^{\epsilon}}|\Upsilon(x, y, t)-\Upsilon(x, x, t)| \rho_{\epsilon}(x-y) d y d x d t
\end{aligned}
$$

On one hand, thanks to the regularity of $f$, the function

$$
v \mapsto \operatorname{sign}(u(x, t)-v)\left(\tilde{\partial}_{x} f(v ; x, t)-\tilde{\partial}_{x} f(u(x, t) ; x, t)\right)
$$

is $\left\|\partial_{s} \partial_{x} f\right\|_{\infty}$-Lipschitz continuous. Therefore, it follows from the definition of $A(x, y, t)$ that

$$
|A(x, y, t)-A(x, x, t)| \leq\left\|\partial_{s} \partial_{x} f\right\|_{\infty}|v(y, t)-v(x, t)| .
$$

Thus, using the fact the both $\rho_{\epsilon}$ and $s \mapsto-s \rho_{\epsilon}^{\prime}(s)$ are approximations of the unit, the relation (86) ensures that

$$
\begin{aligned}
& \int_{0}^{T} \chi(t) \int_{x \in B_{t}} \int_{y \in B_{t}^{\epsilon}} A(x, y, t) \partial_{x}\left[(y-x) \rho_{\epsilon}(x-y)\right] d y d x d t \\
& \leq 2 \epsilon\left\|\partial_{s} \partial_{x} f\right\|_{\infty}\|\chi\|_{\infty} \int_{0}^{T} \operatorname{TV}(v(\cdot, t)) d t .
\end{aligned}
$$

Hence, using (66) provides that

$$
\begin{aligned}
\int_{0}^{T} \chi(t) \int_{x \in B_{t}} \int_{y \in B_{t}^{\epsilon}} A(x, y, t) \partial_{x}\left[(y-x) \rho_{\epsilon}(x-y)\right] d y d x d t \\
\leq 2 \epsilon\left\|\partial_{s} \partial_{x} f\right\|_{\infty}\|\chi\|_{\infty}\left(C_{g, 0}(T) \operatorname{TV}\left(v_{0}\right)+C_{g, 1}(T)\right) .
\end{aligned}
$$

On the other hand, the regularity of $f$ ensures that

$$
|B(x, y, t)-B(x, x, t)| \leq \chi(t) \sup _{s \in \mathbb{R}}\left\|\partial_{x x}^{2} f(s ; \cdot, t)\right\|_{L^{\infty}\left(B_{t}^{\epsilon}\right)}|x-y| .
$$

As a consequence, it follows from (86) that

$$
\begin{array}{r}
\int_{0}^{T} \chi(t) \int_{x \in B_{t}} \int_{y \in B_{t}^{\epsilon}} B(x, y, t) \partial_{x}\left[(y-x) \rho_{\epsilon}(x-y)\right] d y d x d t \\
\leq 2 \epsilon \int_{0}^{T} \chi(t) \sup _{s \in \mathbb{R}}\left\|\partial_{x x}^{2} f(s ; \cdot, t)\right\|_{L^{\infty}\left(B_{t}^{\epsilon}\right)}\left(\int_{B_{t}} d x\right) d t .
\end{array}
$$


Taking (87) and (88) into account in (85) yields

$$
\begin{aligned}
& D_{31}^{\epsilon} \leq 2 \epsilon\|\chi\|_{\infty}\left[\left\|\partial_{s} \partial_{x} f\right\|_{\infty}\left(C_{g, 0}(T) \operatorname{TV}\left(v_{0}\right)+C_{g, 1}(T)\right)\right. \\
&\left.+\int_{0}^{T} \sup _{s \in \mathbb{R}}\left\|\partial_{x x}^{2} f(s ; \cdot, t)\right\|_{L^{\infty}\left(B_{t}^{\epsilon}\right)}\left(\int_{B_{t}} d x\right) d t\right] .
\end{aligned}
$$

The relations (83), (84) and (89) thus provide that

$$
\begin{aligned}
D_{3}^{\epsilon} \leq \epsilon\|\chi\|_{\infty}\left[3 \int_{0}^{T}\right. & \sup _{s \in \mathbb{R}}\left\|\partial_{x x}^{2} f(s, \cdot, t)\right\|_{L^{\infty}\left(B_{t}^{\epsilon}\right)}\left(\int_{B_{t}} d x\right) d t \\
& \left.+2\left\|\partial_{s} \partial_{x} f\right\|_{\infty}\left(C_{g, 0}(T) \operatorname{TV}\left(v_{0}\right)+C_{g, 1}(T)\right)\right] .
\end{aligned}
$$

Concerning $D_{4}^{\epsilon}$, it follows from (68) and from $\int_{\mathbb{R}}\left|\rho_{\epsilon}^{\prime}(x-y)\right| d x=2 / \epsilon$ that

$$
D_{4}^{\epsilon} \geq-\frac{2}{\epsilon}\|\chi\|_{\infty} \int_{0}^{T} \int_{y \in B_{t}^{\epsilon}}\|(f-g)(\cdot ; y, t)\|_{\infty} d y d t .
$$

Thanks to (69), one has

$$
D_{5}^{\epsilon} \geq-\|\chi\|_{\infty} \int_{0}^{T} \int_{y \in B_{t}^{\epsilon}}\left\|\partial_{y}(f-g)(\cdot ; y, t)\right\|_{\infty} d y d t .
$$

By choosing $\chi(t)=\min (1 ; \max (0, \lambda(T-t)))$ and letting $\lambda$ tend to $+\infty$ in $(77)-(79)$, (91) and (92), we obtain that

$$
\int_{B_{T}}|u(x, T)-v(x, T)| d x \leq \int_{B_{0}}\left|u_{0}(x)-v_{0}(x)\right| d x+\epsilon C_{1}^{\epsilon}+\frac{C_{2}^{\epsilon}}{\epsilon}+C_{3}^{\epsilon},
$$

where the quantities $C_{1}^{\epsilon}, C_{2}^{\epsilon}$ and $C_{3}^{\epsilon}$ have been made explicit in Theorem A.3. Since the result above holds for all $\epsilon>0$, it also holds for the optimal choice of $\epsilon$, concluding the proof of Theorem A.3.

Acknowledgments. This work has been supported by the LRC Manon (Modélisation et approximation numérique orientées pour l'énergie nucléaire - CEA/DM2SLJLL) and by the Nuclear System and Scenarios federative project of the NEEDS program (CNRS - CEA - AREVA - EDF - IRSN).

\section{REFERENCES}

[1] R. L. Actis, B. A. Szabo, and C. Schwab. Hierarchic models for laminated plates and shells. Comput. Methods Appl. Mech. Engrg., 172(1-4):79-107, 1999.

[2] C. Bernardi, T. C. Rebollo, F. Hecht, and R. Lewandowski. Automatic insertion of a turbulence model in the finite element discretization of the Navier-Stokes equations. Math. Models Methods Appl. Sci., 19(7):1139-1183, 2009.

[3] F. Bouchut and B. Perthame. Kružkov's estimates for scalar conservation laws revisited. Trans. Amer. Math. Soc., 350(7):2847-2870, 1998.

[4] B. Boutin, F. Coquel, and P. G. LeFloch. Coupling techniques for nonlinear hyperbolic equations. III: A regularization method based on thick interfaces. SIAM J. Numer. Anal., 2013.

[5] M. Braack and A. Ern. A posteriori control of modeling errors and discretization errors. Multiscale Model. Simul., 1(2):221-238 (electronic), 2003.

[6] C. Cancès and T. Gallouët. On the time continuity of entropy solutions. J. Evol. Equ., 11(1):43-55, 2011.

[7] C. Cancès and N. Seguin. Error estimate for Godunov approximation of locally constrained conservation laws. SIAM J. Numer. Anal., 50(6):3036-3060, 2012. 
[8] C. Chainais-Hillairet. Finite volume schemes for a nonlinear hyperbolic equation. Convergence towards the entropy solution and error estimate. M2AN Math. Model. Numer. Anal., 33(1):129-156, 1999.

[9] C. Chainais-Hillairet and S. Champier. Finite volume schemes for nonhomogeneous scalar conservation laws: error estimate. Numer. Math., 88(4):607-639, 2001

[10] B. Cockburn, F. Coquel, and P. G. LeFloch. An error estimate for finite volume methods for multidimensional conservation laws. Math. Comp., 63:77-103, 1994.

[11] R. M. Colombo and M. Garavello. A well posed Riemann problem for the $p$-system at a junction. Netw. Heterog. Media, 1(3):495-511 (electronic), 2006.

[12] R. M. Colombo, M. Mercier, and M. D. Rosini. Stability and total variation estimates on general scalar balance laws. Commun. Math. Sci., 7(1):37-65, 2009.

[13] N. Crouseilles and M. Lemou. An asymptotic preserving scheme based on a micro-macro decomposition for collisional Vlasov equations: diffusion and high-field scaling limits. Kinet. Relat. Models, 4(2):441-477, 2011.

[14] C. M. Dafermos. Hyperbolic conservation laws in continuum physics, volume 325 of Grundlehren der Mathematischen Wissenschaften [Fundamental Principles of Mathematical Sciences]. Springer-Verlag, Berlin, third edition, 2010.

[15] P. Degond, G. Dimarco, and L. Mieussens. A moving interface method for dynamic kineticfluid coupling. J. Comput. Phys., 227(2):1176-1208, 2007.

[16] P. Degond, G. Dimarco, and L. Mieussens. A multiscale kinetic-fluid solver with dynamic localization of kinetic effects. J. Comput. Phys., 229(13):4907-4933, 2010.

[17] R. Eymard, T. Gallouët, M. Ghilani, and R. Herbin. Error estimates for the approximate solutions of a nonlinear hyperbolic equation given by finite volume scheme. IMA J. Numer. Anal., 18(4):563-594, 1998.

[18] E. Godlewski and P.-A. Raviart. The numerical interface coupling of nonlinear hyperbolic systems of conservation laws. I. The scalar case. Numer. Math., 97(1):81-130, 2004.

[19] S. Jin, J.-G. Liu, and L. Wang. A domain decomposition method for semilinear hyperbolic systems with two-scale relaxations. Math. Comp., 82(282):749-779, 2013.

[20] D. Kröner and M. Ohlberger. A posteriori error estimates for upwind finite volume schemes. Math. Comp., 69(229):25-39, 2000.

[21] S. N. Kružkov. First order quasilinear equations with several independent variables. Mat. Sb. (N.S.), 81(123):228-255, 1970.

[22] N. N. Kuznetsov. The accuracy of certain approximate methods for the computation of weak solutions of a first order quasilinear equation. Ž. Vyčisl. Mat. i Mat. Fiz., 16(6):1489-1502, 1627, 1976.

[23] M. Lécureux-Mercier. Improved stability estimates for general scalar conservation laws. $J$. Hyperbolic Differ. Equ., 8(4):727-757, 2011.

[24] B. J. Lucier. A moving mesh numerical method for hyperbolic conservation laws. Math. Comp., 46(173):59-69, 1986.

[25] H. Mathis, C. Cancès, E. Godlewski, and N. Seguin. Model adaptation for hyperbolic systems with relaxation. Submitted for publication, 2013.

[26] L. Mirabella, F. Nobile, and A. Veneziani. An a posteriori error estimator for model adaptivity in electrocardiology. Comput. Methods Appl. Mech. Engrg., 200(37-40):2727-2737, 2011.

[27] J. T. Oden, S. Prudhomme, A. Romkes, and P. T. Bauman. Multiscale modeling of physical phenomena: adaptive control of models. SIAM J. Sci. Comput., 28(6):2359-2389, 2006.

[28] J. T. Oden and K. S. Vemaganti. Estimation of local modeling error and goal-oriented adaptive modeling of heterogeneous materials. I. Error estimates and adaptive algorithms. $J$. Comput. Phys., 164(1):22-47, 2000.

[29] J. T. Oden and T. I. Zohdi. Analysis and adaptive modeling of highly heterogeneous elastic structures. Comput. Methods Appl. Mech. Engrg., 148(3-4):367-391, 1997.

[30] E. Stein and S. Ohnimus. Anisotropic discretization- and model-error estimation in solid mechanics by local Neumann problems. Comput. Methods Appl. Mech. Engrg., 176(1-4):363385, 1999. New advances in computational methods (Cachan, 1997).

[31] K. S. Vemaganti and J. T. Oden. Estimation of local modeling error and goal-oriented adaptive modeling of heterogeneous materials. II. A computational environment for adaptive modeling of heterogeneous elastic solids. Comput. Methods Appl. Mech. Engrg., 190(46-47):6089$6124,2001$. 
[32] T. I. Zohdi, J. T. Oden, and G. J. Rodin. Hierarchical modeling of heterogeneous bodies. Comput. Methods Appl. Mech. Engrg., 138(1-4):273-298, 1996.

Clément CAncÈs, Edwige GodLewski

UPMC Univ Paris 06, UMR 7598, Laboratoire Jacques-Louis Lions, F-75005, Paris, France CNRS, UMR 7598, Laboratoire Jacques-Louis Lions, F-75005, Paris, France

cances@ann.jussieu.fr, edwige.godlewski@upmc.fr

Frédéric COQUEL

CNRS, UMR 7641, CMAP Ecole Polytechnique, Palaiseau, F-91128, France

frederic.coquel@cmap.polytechnique.fr

Hélène MATHIS

Université de Nantes, Laboratoire de Mathématiques Jean Leray, 2, Rue de la Houssinière, 44322 Nantes Cedex 03, France

helene.mathis@univ-nantes.fr

Nicolas SEGUIN

INRIA Rocquencourt, BP 105, F-78153, Le Chesnay Cedex, France

UPMC Univ Paris 06, UMR 7598, Laboratoire Jacques-Louis Lions, F-75005, Paris, France CNRS, UMR 7598, Laboratoire Jacques-Louis Lions, F-75005, Paris, France

nicolas.seguin@upmc.fr 